WUHEP/97-15

\title{
Model of supersymmetric quantum field theory with broken parity symmetry
}

\author{
Carl M. Bender* \\ Department of Physics, Washington University, St. Louis, MO 63130, USA \\ Kimball A. Milton ${ }^{\dagger}$ \\ Department of Physics and Astronomy, University of Oklahoma, Norman, OK 73019, USA
}

(October 20, 2018)

\begin{abstract}
Recently, it was observed that self-interacting scalar quantum field theories having a non-Hermitian interaction term of the form $g(i \phi)^{2+\delta}$, where $\delta$ is a real positive parameter, are physically acceptable in the sense that the energy spectrum is real and bounded below. Such theories possess PT invariance, but they are not symmetric under parity reflection or time reversal separately. This broken parity symmetry is manifested in a nonzero value for $\langle\phi\rangle$, even if $\delta$ is an even integer. This paper extends this idea to a two-dimensional supersymmetric quantum field theory whose superpotential is $\mathcal{S}(\phi)=-i g(i \phi)^{1+\delta}$. The resulting quantum field theory exhibits a broken parity symmetry for all $\delta>0$. However, supersymmetry remains unbroken, which is verified by showing that the ground-state energy density vanishes and that the fermion-boson mass ratio is unity.
\end{abstract}

PACS number(s): 12.60.Jv, 02.30.Mv, 11.30.Er, 11.30.Pb

Typeset using REVTEX

*Electronic address: cmb@howdy.wustl.edu

${ }^{\dagger}$ Electronic address: milton@mail.nhn.ou.edu 


\section{INTRODUCTION}

It is extremely difficult to perform conventional perturbative calculations in supersymmetric field theory because expansions in powers of the coupling constant are infrared divergent. Furthermore, introducing a regulator in the form of a momentum cutoff or a lattice spacing to control this divergence breaks the supersymmetry invariance. An especially simple way to solve this problem is to use the delta expansion, a perturbative expansion in powers of the degree of the nonlinearity of the interaction term [1].

The key idea of the delta expansion is to replace a self-interaction term of the form $g \phi^{4}$ by $g\left(\phi^{2}\right)^{1+\delta}$, where $\delta$ is regarded as a small parameter. Thus, the parameter $\delta$ measures the departure from linearity of the field equation. A graphical procedure for expanding the Green's functions of a quantum field theory in powers of $\delta$ is given in Ref. [1]. The advantage of such an expansion is that it has a nonzero radius of convergence and that it yields numerically accurate nonperturbative information when $\delta$ is set equal to 1 .

The delta expansion is broadly useful for nonlinear problems. It has been applied to many classical nonlinear ordinary differential equations of mathematical physics and it has given superbly accurate numerical results [2]. The delta expansion has also been successfully applied to some nonlinear partial differential equations of mathematical physics [3].

In the context of quantum field theory the delta expansion has been used to study renormalization [4], local gauge invariance [5], stochastic quantization [6], finite-temperature field theory [7], and the Ising limit of quantum field theory [8].

The delta expansion is particularly well suited for studying supersymmetric models of quantum field theory because expansions in powers of $\delta$ are not infrared divergent and the delta expansion respects the supersymmetry exactly. Calculations to second order in powers of $\delta$ have been done for the ground-state energy [9] and the fermion-boson mass ratio [10].

Recently, we have examined a new class of scalar quantum field theories that are not symmetric under parity reflection [11]. The Euclidean-space Lagrangian for this class of theories is

$$
\mathcal{L}=\frac{1}{2}(\partial \phi)^{2}+\frac{1}{2} m^{2} \phi^{2}-g(i \phi)^{2+\delta} \quad(\delta>-2) .
$$

The Hamiltonian for such theories is not Hermitian. However, there is strong evidence that these theories possess energy spectra that are real and bounded below. The theories in

\footnotetext{
${ }^{1}$ There is copious numerical evidence that such theories possess positive spectra when $m \neq 0$. Furthermore, one can understand positivity from a theoretical point of view. Consider, for example, the weak-coupling expansion for the $d$-dimensional Euclidean quantum field theory defined by the Lagrangian $\mathcal{L}=\frac{1}{2}(\partial \phi)^{2}+\frac{1}{2} m^{2} \phi^{2}+g i \phi^{3}$. For a conventional $g \phi^{3}$ theory the weak-coupling expansion is real, and (apart from a possible overall factor of $g$ ) the Green's functions are formal power series in $g^{2}$. These series are not Borel summable because they do not alternate in sign. Nonsummability reflects the fact that the spectrum of the underlying theory is not bounded below. However, when we replace $g$ by $i g$, the perturbation series remains real but now alternates in sign. Thus, the perturbation series is now summable and this suggests that the underlying theory has a real positive spectrum.
} 
Eq. (1.1) are a natural field theoretic generalization of a remarkable quantum mechanical Hamiltonian studied by D. Bessis and C. Itzykson: [12

$$
H=\frac{1}{2} p^{2}+i x^{3}
$$

The Lagrangian in Eq. (1.1) is intriguing because it is not parity symmetric. This is manifested by a nonzero value of $\langle\phi\rangle$. It is interesting that this broken symmetry persists even when $\delta$ is an even integer [11].

That fact that the Lagrangian in Eq. (1.1) has a broken parity symmetry suggests that if we construct a two-dimensional supersymmetric quantum field theory by using a superpotential of the form

$$
\mathcal{S}(\phi)=-i g(i \phi)^{1+\delta}
$$

the resulting theory will also have a broken parity symmetry. The supersymmetric Lagrangian resulting from the superpotential (1.3) is

$$
\begin{aligned}
\mathcal{L} & =\frac{1}{2}(\partial \phi)^{2}+\frac{1}{2} i \bar{\psi} \not \partial \psi+\frac{1}{2} \mathcal{S}^{\prime}(\phi) \bar{\psi} \psi+\frac{1}{2}[\mathcal{S}(\phi)]^{2} \\
& =\frac{1}{2}(\partial \phi)^{2}+\frac{1}{2} i \bar{\psi} \not \partial \psi+\frac{1}{2} g(1+\delta)(i \phi)^{\delta} \bar{\psi} \psi-\frac{1}{2} g^{2}(i \phi)^{2+2 \delta}
\end{aligned}
$$

where $\psi$ is a Majorana spinor.

The Lagrangian (1.4) raises an interesting question. Will the breaking of parity symmetry induce a breaking of supersymmetry? To answer this question we calculate both the groundstate energy $E_{\text {ground state }}$ and the fermion-boson mass ratio $R$ as series in powers of the parameter $\delta$. We find that through second order in $\delta, E_{\text {ground state }}=0$ and $R=1$, which strongly suggests that supersymmetry remains unbroken. Based on the experience with these calculations we believe that our results are valid to all orders in powers of $\delta$. It is quite difficult to break supersymmetry [13.

This paper is organized very simply. In Sec. II we explain our calculational procedure. We derive a set of Feynman rules for obtaining the Green's functions to a given order in powers of $\delta$ for the scalar field theory Lagrangian (1.1). Next, in Sec. III we apply the procedures of Sec. III to calculate the ground-state energy and the fermion-boson mass ratio to first order in $\delta$ for the supersymmetric Lagrangian (1.4). In Sec. IV we perform these calculations to second order in $\delta$. Finally, in Sec. $\nabla$ we examine some of the formal cancellations that occur in our calculations to see if there could be anomalous contributions to the ground-state energy.

\section{DELTA EXPANSION FOR A PARITY-VIOLATING SCALAR FIELD THEORY}

In this section we explain how to calculate the Green's functions for the scalar quantum field theory described by the Lagrangian $\mathcal{L}$ in Eq. (1.1). Specifically, we follow the procedures described in Ref. [1] and determine a set of graphical rules for constructing the delta expansion through second order in powers of $\delta$. These rules consist of the amplitudes for 
the vertices and lines of a related Lagrangian in which the boson fields are raised to integer powers.

We begin by expanding the Lagrangian $\mathcal{L}$ in Eq. (1.1) to second order in powers of $\delta$ :

$$
\mathcal{L}=\frac{1}{2}(\partial \phi)^{2}+\frac{1}{2} m^{2} \phi^{2}+g \phi^{2}+\delta g \phi^{2} \ln (i \phi)+\frac{1}{2} \delta^{2} g \phi^{2}[\ln (i \phi)]^{2}+\mathrm{O}\left(\delta^{3}\right) .
$$

Observe that at $\delta=0$ the Lagrangian contains the coupling $g$ :

$$
\mathcal{L}_{0}=\frac{1}{2}(\partial \phi)^{2}+\frac{1}{2} m^{2} \phi^{2}+g \phi^{2}
$$

Thus, an expansion in powers of $\delta$ is clearly nonperturbative in $g$.

In general, the functional integral representation for an $n$-point correlation function has the form (modulo the usual normalization factor)

$$
\left\langle 0\left|\phi\left(x_{1}\right) \phi\left(x_{2}\right) \phi\left(x_{3}\right) \ldots \phi\left(x_{n}\right)\right| 0\right\rangle=\int D \phi \phi\left(x_{1}\right) \phi\left(x_{2}\right) \phi\left(x_{3}\right) \ldots \phi\left(x_{n}\right) e^{-\int d x \mathcal{L}} .
$$

We expand the exponential in the integrand in Eq. (2.3) and obtain

$$
e^{-\int d x \mathcal{L}}=e^{-\int d x \mathcal{L}_{0}} \mathcal{E}[\phi]
$$

where

$$
\begin{gathered}
\mathcal{E}[\phi]=1-\delta \\
\delta \int d x \phi^{2} \ln (i \phi)-\frac{1}{2} \delta^{2} g \int d x \phi^{2}[\ln (i \phi)]^{2} \\
+\frac{1}{2} \delta^{2} g^{2}\left[\int d x \phi^{2} \ln (i \phi)\right]^{2}+\mathrm{O}\left(\delta^{3}\right) .
\end{gathered}
$$

Next, we use the identity

$$
\ln (i \phi)=\ln (|\phi|)+\frac{1}{2} i \pi \frac{\phi}{|\phi|},
$$

where $\phi /|\phi|$ represents the algebraic sign of $\phi$, to decompose the expression for $\mathcal{E}$ in (2.5) into its real and imaginary parts:

$$
\begin{aligned}
\mathcal{E}[\phi]=1 & -\delta g \int d x \phi^{2} \ln (|\phi|)-\frac{1}{2} i \pi \delta g \int d x \phi|\phi|-\frac{1}{2} \delta^{2} g \int d x \phi^{2}[\ln (|\phi|)]^{2} \\
& -\frac{1}{2} i \pi \delta^{2} g \int d x \phi|\phi| \ln (|\phi|)+\frac{1}{8} \pi^{2} \delta^{2} g \int d x \phi^{2}+\frac{1}{2} \delta^{2} g^{2}\left[\int d x \phi^{2} \ln (|\phi|)\right]^{2} \\
& +\frac{1}{2} i \pi \delta^{2} g^{2} \int d x \phi^{2} \ln (|\phi|) \int d x \phi|\phi|-\frac{1}{8} \pi^{2} \delta^{2} g^{2}\left[\int d x \phi|\phi|\right]^{2}+\mathrm{O}\left(\delta^{3}\right) .
\end{aligned}
$$

It is easy to construct an effective Lagrangian $\tilde{\mathcal{L}}$ having polynomial interaction terms that can be used to calculate the Green's functions of (2.7) to first order in $\delta$ :

$$
\tilde{\mathcal{L}}=\mathcal{L}_{0}+\frac{1}{2} \delta g \phi^{2 \alpha+2}+\frac{1}{2} i \pi \delta g \alpha \phi^{2 \alpha+2 \gamma+1}
$$


To use this Lagrangian we must assume that the parameters $\alpha$ and $\gamma$ are integer. We can then read off a set of Feynman amplitudes:

$$
\begin{aligned}
\text { boson line: } & \frac{1}{p^{2}+m^{2}+2 g}, \\
(2 \alpha+2) \text { boson vertex: } & -\frac{1}{2} \delta g(2 \alpha+2) ! \\
(2 \alpha+2 \gamma+1) \text { boson vertex: } & -\frac{1}{2} i \pi \delta g \alpha(2 \alpha+2 \gamma+1) ! .
\end{aligned}
$$

Note that the vertices are of order $\delta$. Thus, if we are calculating to first order in $\delta$ we need only include graphs having one vertex. Once a graphical calculation has been completed we then apply the derivative operator

$$
\mathcal{D}=\frac{\partial}{\partial \alpha}
$$

followed by setting $\alpha=0$ and $\gamma=\frac{1}{2}$. The technique of using a derivative operator to recover the Green's functions for the Lagrangian (1.1) is like the replica trick used in calculations in statistical mechanical models. It is a standard procedure used in all papers on the delta expansion and is is discussed in great detail in Refs. [1, 9, 10].

To perform calculations to second order in $\delta$ we seek a higher-order effective Lagrangian $\tilde{\mathcal{L}}$ of the form

$$
\begin{aligned}
\tilde{\mathcal{L}}=\mathcal{L}_{0} & +\left(\delta A_{1}+\delta^{2} A_{2}\right) g \phi^{2 \alpha+2}+\left(\delta B_{1}+\delta^{2} B_{2}\right) g \phi^{2 \beta+2} \\
& +\left(\delta C_{1}+\delta^{2} C_{2}\right) g \phi^{2 \alpha+2 \gamma+1}+\left(\delta D_{1}+\delta^{2} D_{2}\right) g \phi^{2 \beta+2 \gamma+1}
\end{aligned}
$$

To determine the coefficients $A_{1}, A_{2}, B_{1}, B_{2}, C_{1}, C_{2}, D_{1}$, and $D_{2}$, we replace $\mathcal{L}$ by $\tilde{\mathcal{L}}$ on the left side of Eq. (2.4) and expand to second order in $\delta$. We then apply the derivative operator [1]

$$
\mathcal{D}=\frac{1}{2}\left(\frac{\partial}{\partial \alpha}-\frac{\partial}{\partial \beta}\right)+\frac{1}{4}\left(\frac{\partial^{2}}{\partial \alpha^{2}}+\frac{\partial^{2}}{\partial \beta^{2}}\right)
$$

and set $\alpha=0, \beta=0$, and $\gamma=\frac{1}{2}$. By comparing with the right side of Eq. (2.7) we obtain a set of seven simultaneous equations for the coefficients:

$$
\begin{aligned}
& \left.\mathcal{D}\left(A_{1} \int d x \phi^{2 \alpha+2}+B_{1} \int d x \phi^{2 \beta+2}\right)\right|_{\substack{\alpha=\beta=0 \\
\gamma=\frac{1}{2}}}=\int d x \phi^{2} \ln (|\phi|), \\
& \left.\mathcal{D}\left(A_{2} \int d x \phi^{2 \alpha+2}+B_{2} \int d x \phi^{2 \beta+2}\right)\right|_{\substack{\alpha=\beta=0 \\
\gamma=\frac{1}{2}}}=\frac{1}{2} \int d x \phi^{2}[\ln (|\phi|)]^{2}-\frac{1}{8} \pi^{2} \int d x \phi^{2}, \\
& \left.\mathcal{D}\left(C_{1} \int d x \phi^{2 \alpha+2 \gamma+1}+D_{1} \int d x \phi^{2 \beta+2 \gamma+1}\right)\right|_{\substack{\alpha=\beta=0 \\
\gamma=\frac{1}{2}}}=\frac{1}{2} i \pi \int d x \phi|\phi|, \\
& \left.\mathcal{D}\left(C_{2} \int d x \phi^{2 \alpha+2 \gamma+1}+D_{2} \int d x \phi^{2 \beta+2 \gamma+1}\right)\right|_{\substack{\alpha=\beta=0 \\
\gamma=\frac{1}{2}}}=\frac{1}{2} i \pi \int d x \phi|\phi| \ln (|\phi|),
\end{aligned}
$$




$$
\begin{gathered}
\left.\mathcal{D}\left(A_{1} \int d x \phi^{2 \alpha+2}+B_{1} \int d x \phi^{2 \beta+2}\right)^{2}\right|_{\substack{\alpha=\beta=0 \\
\gamma=\frac{1}{2}}}=\left[\int d x \phi^{2} \ln (|\phi|)\right]^{2}, \\
\left.\mathcal{D}\left(C_{1} \int d x \phi^{2 \alpha+2 \gamma+1}+D_{1} \int d x \phi^{2 \beta+2 \gamma+1}\right)^{2}\right|_{\substack{\alpha=\beta=0 \\
\gamma=\frac{1}{2}}}=-\frac{1}{4} \pi^{2}\left(\int d x \phi|\phi|\right)^{2}, \\
\mathcal{D}\left(A_{1} C_{1} \int d x \phi^{2 \alpha+2} \int d x \phi^{2 \alpha+2 \gamma+1}+A_{1} D_{1} \int d x \phi^{2 \alpha+2} \int d x \phi^{2 \beta+2 \gamma+1}\right. \\
\left.+B_{1} C_{1} \int d x \phi^{2 \beta+2} \int d x \phi^{2 \alpha+2 \gamma+1}+B_{1} D_{1} \int d x \phi^{2 \beta+2} \int d x \phi^{2 \beta+2 \gamma+1}\right)\left.\right|_{\substack{\alpha=\beta=0 \\
\gamma=\frac{1}{2}}} \\
=\frac{1}{2} i \pi \int d x \phi^{2} \ln (|\phi|) \int d x \phi|\phi| .
\end{gathered}
$$

The solution to these equations is:

$$
\begin{aligned}
A_{1} & =\frac{1}{2}, \\
B_{1} & =-\frac{1}{2}, \\
C_{1} & =\frac{1}{2} i \pi \alpha, \\
D_{1} & =-\frac{1}{2} i \pi \beta, \\
A_{2} & =\frac{1}{4}-\frac{1}{8} \pi^{2} \alpha^{2}, \\
B_{2} & =\frac{1}{4}-\frac{1}{8} \pi^{2} \beta^{2}, \\
C_{2} & =\frac{1}{4} i \pi, \\
D_{2} & =-\frac{1}{4} i \pi .
\end{aligned}
$$

We thus read off the Feynman amplitudes for the effective Lagrangian $\tilde{\mathcal{L}}$ to second order in $\delta$ :

$$
\begin{aligned}
\text { boson line: } & \frac{1}{p^{2}+m^{2}+2 g}, \\
(2 \alpha+2) \text { boson vertex: } & \left(-\frac{1}{2} \delta-\frac{1}{4} \delta^{2}+\frac{1}{8} \pi^{2} \delta^{2} \alpha^{2}\right) g(2 \alpha+2) !, \\
(2 \beta+2) \text { boson vertex: } & \left(\frac{1}{2} \delta-\frac{1}{4} \delta^{2}+\frac{1}{8} \pi^{2} \delta^{2} \beta^{2}\right) g(2 \beta+2) !, \\
(2 \alpha+2 \gamma+1) \text { boson vertex: } & \left(-\frac{1}{2} i \pi \delta \alpha-\frac{1}{4} i \pi \delta^{2}\right) g(2 \alpha+2 \gamma+1) !, \\
(2 \beta+2 \gamma+1) \text { boson vertex: } & \left(\frac{1}{2} i \pi \delta \beta+\frac{1}{4} i \pi \delta^{2}\right) g(2 \beta+2 \gamma+1) !
\end{aligned}
$$


To second order in $\delta$ one must include all graphs containing up to two vertices and treat the parameters $\alpha, \beta$, and $\gamma$ as integers. At the end of the calculation one must then apply the derivative operator $\mathcal{D}$ in Eq. (2.12) and set $\alpha=0, \beta=0, \gamma=\frac{1}{2}$.

In the next two sections we generalize this approach to the case of supersymmetric Lagrangians, identify the Feynman rules for calculating Green's functions, and use these rules to calculate the ground-state energy, the one-point Green's function, and the fermionboson mass ratio.

\section{FIRST-ORDER CALCULATIONS FOR THE SUPERSYMMETRIC LAGRANGIAN}

In this section we show how to calculate to first order in $\delta$ the ground-state energy and the fermion-boson mass ratio for the two-dimensional Euclidean field theory defined by Eq. (1.4). We follow the procedure described in Sec. II and obtain a set of graphical rules for constructing the delta expansion to a given order in powers of $\delta$. These rules are the amplitudes for vertices and lines of a related effective Lagrangian in which the boson fields are raised to integer powers.

Our first objective is to obtain this related Lagrangian. We begin by expanding Eq. (11.4) to first order in $\delta$ :

$$
\begin{aligned}
\mathcal{L}=\frac{1}{2}(\partial \phi)^{2} & +\frac{1}{2} g^{2} \phi^{2}+\frac{1}{2} i \bar{\psi} \not \partial \psi+\frac{1}{2} g \bar{\psi} \psi \\
& +\delta g^{2} \phi^{2} \ln (i \phi)+\frac{1}{2} \delta g \bar{\psi} \psi+\frac{1}{2} \delta g \bar{\psi} \psi \ln (i \phi)+\mathrm{O}\left(\delta^{2}\right) .
\end{aligned}
$$

We can display the real and imaginary parts of this Lagrangian explicitly by using the identity (2.6).

Unfortunately, the Lagrangian (3.1) is nonpolynomial so we cannot read off a conventional set of Feynman rules. However, consider the following effective Lagrangian whose interaction terms are polynomial in form:

$$
\begin{aligned}
\tilde{\mathcal{L}}= & \frac{1}{2}(\partial \phi)^{2}+\frac{1}{2} g^{2} \phi^{2}+\frac{1}{2} i \bar{\psi} \not \partial \psi+\frac{1}{2} g \bar{\psi} \psi \\
& +\frac{1}{2} \delta g^{2} \phi^{2+2 \alpha}+\frac{1}{4} \delta(2 \alpha+1) g \bar{\psi} \psi \phi^{2 \alpha}+\frac{1}{2} i \pi \delta g^{2} \alpha \phi^{2 \alpha+2 \gamma+1}+\frac{1}{4} i \pi \delta g \alpha \bar{\psi} \psi \phi^{2 \alpha+2 \gamma-1}
\end{aligned}
$$

where $\alpha$ and $\gamma$ are to be regarded temporarily as positive integers. Note that we recover the Lagrangian $\mathcal{L}$ in Eq. (3.1) if we apply the derivative operator $\mathcal{D}$ in Eq. (2.10) to $\tilde{\mathcal{L}}$ and set $\alpha=0$ and $\gamma=\frac{1}{2}$.

To calculate the Green's functions for $\mathcal{L}$ we use the same device. That is, we find the Green's functions for $\tilde{\mathcal{L}}$. We then apply $\mathcal{D}$ to these Green's functions and set $\alpha=0$ and

$\gamma=\frac{1}{2}$. To calculate the Green's functions for $\tilde{\mathcal{L}}$ we read off the following Feynman rules from Eq. (3.2):

$$
\text { boson line: } \quad \tilde{\Delta}(p)=\frac{1}{p^{2}+g^{2}}
$$




$$
\begin{array}{cl}
\text { fermion line: } & \tilde{S}(p)=\frac{1}{g-\not p}, \\
(2 \alpha+2) \text { boson vertex: } & -\frac{1}{2} \delta g^{2}(2 \alpha+2) ! \\
& =\frac{1}{2} \delta g(2 \alpha+1) ! \\
(2 \alpha) \text { boson and } 2 \text { fermion vertex: } & -\frac{1}{2} i \pi \delta g^{2} \alpha(2 \alpha+2 \gamma+1) !, \\
(2 \alpha+2 \gamma+1) \text { boson vertex: } & -\frac{1}{2} i \pi \delta g \alpha(2 \alpha+2 \gamma-1) !
\end{array}
$$

Note that these rules are nonperturbative in the coupling constant $g$; the parameter $g$ appears nontrivially in all the vertex and line amplitudes.

In addition to these Feynman rules one must remember to associate a factor of -1 with every fermion loop, and that in two-dimensional space gamma matrices are two dimensional so that the trace of the unit matrix introduces a factor of two. Moreover, one must be careful to associate with each graph the appropriate symmetry number. The fermions for this quantum field theory are Majorana fermions, which are nondirectional. Thus, for example, the symmetry number of a fermion self-loop consisting of a single fermion propagator is $\frac{1}{2}$.

Using these graphical rules, we calculate below the zero-point, one-point, and two-point Green's functions to order $\delta$. Because we are calculating to first order in $\delta$ and all vertices in Eq. (3.3) are of order $\delta$, we need only consider single-vertex graphs. We will show that to order $\delta$ the ground-state energy vanishes and that the renormalized fermion and boson masses are equal. This indicates that the theory is supersymmetric to this order. We also see that the one-point Green's function is nonvanishing, which shows that parity is broken to order $\delta$. These results are obtained using a formal manipulation of divergent quantities in the form of logarithmically divergent integrals. In Sec. V we re-examine these formal manipulations more carefully to see whether an anomalous structure could be present.

\section{A. Calculation of the ground-state energy}

The ground-state energy of a quantum field theory is the negative sum of the connected graphs having no external lines. Because we are considering graphs with no external lines, only the vertices in Eq. (3.3) having an even number of boson lines contribute to the groundstate energy. Our results can be expressed in terms of a single dimensionless divergent integral $\Lambda$, which represents the amplitude of a boson self-loop (a loop formed from one boson propagator):

$$
\Lambda=\Delta(0)=\frac{1}{(2 \pi)^{2}} \int d^{2} p \frac{1}{p^{2}+g^{2}}
$$

where $\Delta(x)$ is the boson propagator in coordinate space [the Fourier transform of $\tilde{\Delta}(p)$ ]. The amplitude for a fermion self-loop can also be expressed in terms of $\Lambda$ :

$$
\frac{1}{(2 \pi)^{2}} \int d^{2} p \operatorname{Tr}\left(\frac{1}{g-\not \partial}\right)=\frac{1}{(2 \pi)^{2}} \int d^{2} p \operatorname{Tr}\left(\frac{g+\not p}{p^{2}+g^{2}}\right)=2 g \Lambda .
$$


Two graphs of order $\delta$ contribute to the ground-state energy density. These graphs are shown in Fig. 1. The amplitudes for these graphs are the product of the vertex and line amplitudes multiplied by the appropriate symmetry numbers. The first graph is a pure boson graph consisting of one vertex with $\alpha+1$ boson self-loops attached. The amplitude for the vertex is $-\frac{1}{2} \delta g^{2}(2 \alpha+2)$ !, the symmetry number for this graph is $2^{-\alpha-1} /(\alpha+1)$ !, the Feynman integral for the graph is $\Lambda^{\alpha+1}$, and we include a factor of -1 because we are calculating the ground-state energy density. The second graph is a mixed boson-fermion graph consisting of one vertex with $\alpha$ boson self-loops and one fermion self-loop attached. The amplitude for the vertex is $-\frac{1}{2} \delta g(2 \alpha+1)$ !, the symmetry number for the graph is $2^{-\alpha-1} / \alpha$ !, the Feynman integral for the graph is $2 g \Lambda^{\alpha+1}$, and there are two factors of -1 , the first because there is a fermion loop and the second because we are calculating the ground-state energy density. In summary, our results are

$$
\begin{aligned}
& \text { Fig. 1a: } \quad \delta g^{2} \frac{(2 \alpha+1) ! \Lambda^{\alpha+1}}{2^{\alpha+1} \alpha !}, \\
& \text { Fig. 1b: } \quad-\delta g^{2} \frac{(2 \alpha+1) ! \Lambda^{\alpha+1}}{2^{\alpha+1} \alpha !} .
\end{aligned}
$$

Observe that the two amplitudes above are equal in magnitude but opposite in sign. Thus, the final result for the ground-state energy to order $\delta$ appears to be 0 . This explicit cancellation holds for all values of $\alpha$; although we expected to differentiate with respect to $\alpha$ and set $\alpha=0$, there seems to be no need to do so in this case and we obtain directly

$$
E_{\text {ground state }}=0+\mathrm{O}\left(\delta^{2}\right)
$$

Of course, the cancellation that leads to this result comes from subtracting two logarithmically divergent integrals. One may ask whether this cancellation persists if these integrals are properly regulated. We examine this question in Sec. V.

\section{B. Calculation of $\langle\phi\rangle$}

In contrast, the one-point Green's function arises only from vertices in Eq. (3.3) having an odd number of boson lines. The two contributing graphs are shown in Fig. 2. The amplitudes are

$$
\begin{aligned}
& \text { Fig. 2a: } \quad-i \pi \delta \alpha \frac{(2 \alpha+2 \gamma+1) ! \Lambda^{\alpha+\gamma}}{2^{\alpha+\gamma+1}(\alpha+\gamma) !} \\
& \text { Fig. 2b: } \quad i \pi \delta \alpha \frac{(2 \alpha+2 \gamma-1) ! \Lambda^{\alpha+\gamma}}{2^{\alpha+\gamma}(\alpha+\gamma-1) !}
\end{aligned}
$$

Adding these amplitudes, differentiating with respect to $\alpha$, and setting $\alpha=0$ and $\gamma=\frac{1}{2}$, we obtain a nonzero result for the vacuum expectation value of the scalar field:

$$
\langle\phi\rangle=G_{1}=-i \delta \sqrt{\pi \Lambda / 2}+\mathrm{O}\left(\delta^{2}\right)
$$

We conclude that this supersymmetric theory has a broken parity symmetry. 
On the basis of calculations performed in Ref. [11], we believe that $\langle\phi\rangle$ remains nonzero even when $\delta$ is a positive integer. One might think that the Lagrangian (1.4) is surely parity symmetric when $\delta$ is an even integer and one might worry the theory does not exist when $\delta$ is an odd integer [because the term $-(i \phi)^{2 \delta+2}$ appears to be unbounded below]. However, neither of these concerns is realized. The reason is that as $\delta$ increases from 0 (free field theory) the entire theory must be analytically continued as a function of $\delta$. The boundary conditions on the functional integral $Z=\int D \phi \exp \left(-\int d x \mathcal{L}\right)$ representing the vacuum persistence function rotate into the complex- $\phi$ plane and yield a broken-parity theory that exists for all $\delta$. This analytic continuation of boundary conditions is discussed in great detail in Ref. [11]. These arguments are based on analysis given in Ref. [14].

\section{Calculation of the fermion-boson mass ratio}

Next, we calculate the mass renormalization of the fermion and the boson. These mass shifts are obtained by evaluating the negative amputated one-particle-irreducible graphs

representing to the two-point Green's functions. One graph contributes to the mass shift of the fermion to order $\delta$ and two graphs contribute to the mass shift of the boson to order $\delta$. These graphs are shown in Fig. 3. The symmetry number for each graph is indicated in the figure. The negative amplitude for the fermion graph is

$$
\text { Fig. 3a: } \delta g \frac{(2 \alpha+1) ! \Lambda^{\alpha}}{2^{\alpha+1} \alpha !}
$$

The negative amplitudes for the boson graphs are

$$
\begin{aligned}
& \text { Fig. 3b: } \quad \delta g^{2} \frac{(2 \alpha+2) ! \Lambda^{\alpha}}{2^{\alpha+1} \alpha !} \\
& \text { Fig. 3c: } \quad-\delta g^{2} \frac{(2 \alpha+1) ! \Lambda^{\alpha}}{2^{\alpha}(\alpha-1) !} .
\end{aligned}
$$

Based on our calculational procedure, the next step would be to differentiate each of these amplitudes with respect to $\alpha$ and set $\alpha=0$. However, this is in fact not even necessary. For arbitrary $\alpha$ the fermion mass is

$$
M_{\text {fermion }}=g+\delta g \frac{(2 \alpha+1) ! \Lambda^{\alpha}}{2^{\alpha+1} \alpha !}+\mathrm{O}\left(\delta^{2}\right)
$$

and the boson mass squared is

$$
M_{\mathrm{boson}}^{2}=g^{2}+\delta g \frac{(2 \alpha+1) ! \Lambda^{\alpha}}{2^{\alpha} \alpha !}+\mathrm{O}\left(\delta^{2}\right)
$$

Thus, to order $\delta$ the fermion-boson mass ratio $R$ is unity:

$$
R=\frac{M_{\text {fermion }}}{M_{\text {boson }}}=1+\mathrm{O}\left(\delta^{2}\right) .
$$

We conclude from these calculations that while parity symmetry is clearly broken, the supersymmetry remains to order $\delta$. In the next section we pursue these calculations to second order in $\delta$. 


\section{SECOND-ORDER CALCULATIONS FOR THE SUPERSYMMETRIC LAGRANGIAN}

In this section we extend the calculations of the previous section to second order in $\delta$. We begin by expanding the Lagrangian (1.4) to order $\delta^{2}$ :

$$
\begin{aligned}
\mathcal{L}=\frac{1}{2}(\partial \phi)^{2} & +\frac{1}{2} g^{2} \phi^{2}+\frac{1}{2} i \bar{\psi} \not \partial \psi+\frac{1}{2} g \bar{\psi} \psi+\frac{1}{2} \delta g \bar{\psi} \psi \\
& +\delta g^{2} \phi^{2} \ln (i \phi)+\frac{1}{2} \delta g \bar{\psi} \psi \ln (i \phi)+\delta^{2} g^{2} \phi^{2}[\ln (i \phi)]^{2} \\
& +\frac{1}{4} \delta^{2} g \bar{\psi} \psi[\ln (i \phi)]^{2}+\frac{1}{2} \delta^{2} g \bar{\psi} \psi \ln (i \phi)+\mathrm{O}\left(\delta^{3}\right)
\end{aligned}
$$

Next we substitute the identity (2.6) and identify a polynomial Lagrangian that in combination with the derivative operator (2.12) gives the Green's functions of the theory defined by the Lagrangian (4.1) to order $\delta^{2}$ :

$$
\begin{aligned}
\tilde{\mathcal{L}}= & \frac{1}{2}(\partial \phi)^{2}+\frac{1}{2} g^{2} \phi^{2}+\frac{1}{2} i \bar{\psi} \not \partial \psi+\frac{1}{2} g \bar{\psi} \psi \\
& +\frac{1}{4}\left(2 \delta+2 \delta^{2}-\pi^{2} \delta^{2} \alpha^{2}\right) g^{2} \phi^{2+2 \alpha}-\frac{1}{4}\left(2 \delta-2 \delta^{2}+\pi^{2} \delta^{2} \beta^{2}\right) g^{2} \phi^{2+2 \beta} \\
& +\frac{1}{16}\left(4 \delta+8 \delta \alpha+2 \delta^{2}+4 \delta^{2} \alpha-\pi^{2} \delta^{2} \alpha^{2}\right) g \bar{\psi} \psi \phi^{2 \alpha} \\
& -\frac{1}{16}\left(4 \delta+8 \delta \beta-2 \delta^{2}-4 \delta^{2} \beta+\pi^{2} \delta^{2} \beta^{2}\right) g \bar{\psi} \psi \phi^{2 \beta} \\
& +\frac{1}{2} i \pi\left(\delta \alpha+\delta^{2}\right) g^{2} \phi^{2 \alpha+2 \gamma+1}-\frac{1}{2} i \pi\left(\delta \beta+\delta^{2}\right) g^{2} \phi^{2 \beta+2 \gamma+1} \\
& +\frac{1}{8} i \pi\left(2 \delta \alpha+\delta^{2} \alpha+2 \delta^{2} \alpha^{2}\right) g \bar{\psi} \psi \phi^{2 \alpha+2 \gamma-1}-\frac{1}{8} i \pi\left(2 \delta \beta-\delta^{2} \beta-2 \delta^{2} \beta^{2}\right) g \bar{\psi} \psi \phi^{2 \beta+2 \gamma-1}
\end{aligned}
$$

To find this effective Lagrangian requires considerable algebra; one must solve a system of eighteen simultaneous equations similar in form to those in Eq. (2.13).

In this effective Lagrangian we treat the parameters $\alpha, \beta$, and $\gamma$ as positive integers so that we can derive a set of Feynman rules for calculating the Green's functions. After these Green's functions have been calculated to second order in $\delta$ we apply the derivative operator $\mathcal{D}$ in Eq. (2.12) and set $\alpha=0, \beta=0$, and $\gamma=\frac{1}{2}$.

The Feynman rules for the Lagrangian (4.2) are the generalization of the rules in Eq. (3.3) to second-order in $\delta$ :

$$
\begin{aligned}
& \text { boson line: } \frac{1}{p^{2}+g^{2}}, \\
& \text { fermion line: } \frac{1}{g-\not p} \\
&(2 \alpha+2) \text { boson vertex: } v_{1}=\frac{1}{4}\left(-2 \delta-2 \delta^{2}+\pi^{2} \delta^{2} \alpha^{2}\right) g^{2}(2 \alpha+2) ! \\
&(2 \beta+2) \text { boson vertex: } v_{2}=\frac{1}{4}\left(2 \delta-2 \delta^{2}+\pi^{2} \delta^{2} \beta^{2}\right) g^{2}(2 \beta+2) !
\end{aligned}
$$


(2 $\alpha$ ) boson and 2 fermion vertex: $v_{3}=\frac{1}{8}\left[-(4+8 \alpha) \delta-\left(2+4 \alpha-\pi^{2} \alpha^{2}\right) \delta^{2}\right] g(2 \alpha)$ !,

$(2 \beta)$ boson and 2 fermion vertex: $v_{4}=\frac{1}{8}\left[(4+8 \beta) \delta-\left(2+4 \beta-\pi^{2} \beta^{2}\right) \delta^{2}\right] g(2 \beta)$ !,

$$
(2 \alpha+2 \gamma+1) \text { boson vertex: } v_{5}=\frac{i \pi}{2}\left(-\delta \alpha-\delta^{2}\right) g^{2}(2 \alpha+2 \gamma+1) !
$$$$
(2 \beta+2 \gamma+1) \text { boson vertex: } v_{6}=\frac{i \pi}{2}\left(\delta \beta+\delta^{2}\right) g^{2}(2 \beta+2 \gamma+1) !
$$

$(2 \alpha+2 \gamma-1)$ boson and 2 fermion vertex: $v_{7}=\frac{i \pi \alpha}{4}\left[-2 \delta-(1+2 \alpha) \delta^{2}\right] g(2 \alpha+2 \gamma-1)$ !,

$(2 \beta+2 \gamma-1)$ boson and 2 fermion vertex: $v_{8}=\frac{i \pi \beta}{4}\left[2 \delta-(1+2 \beta) \delta^{2}\right] g(2 \beta+2 \gamma-1) !$

\section{A. Calculation of the ground-state energy to second order in $\delta$}

There are thirty graphs that contribute to the ground-state energy density. These graphs are organized into seven classes for which the amplitudes combine in a natural way. First, we examine the four single-vertex graphs that are constructed from vertices $v_{1}, v_{2}, v_{3}$, and $v_{4}$. These graphs are shown in Fig. 4(a). The sum of the amplitudes for these graphs is

$$
A_{1}=-\frac{v_{1} \Lambda^{\alpha+1}}{2^{\alpha+1}(\alpha+1) !}-\frac{v_{2} \Lambda^{\beta+1}}{2^{\beta+1}(\beta+1) !}+\frac{v_{3} g \Lambda^{\alpha+1}}{2^{\alpha} \alpha !}+\frac{v_{4} g \Lambda^{\beta+1}}{2^{\beta} \beta !} .
$$

When these amplitudes are combined we find that the contribution of order $\delta$ is identically 0 . Only terms of order $\delta^{2}$ survive. Simplifying the expression in Eq. (4.4) gives

$$
A_{1}=\frac{\delta^{2} g^{2} \Lambda^{\alpha+1}(2 \alpha) !}{2^{\alpha+3} \alpha !}\left[4 \alpha+2-\pi^{2} \alpha^{2}(4 \alpha+1)\right]+(\alpha \rightarrow \beta) .
$$

Next, we consider the two-vertex graphs constructed from $v_{1}$ and $v_{3}$, which are shown in Fig. 4(b). Let $2 l$ be the number of boson lines connecting these two vertices. Then, for each value of $l$ the sum of the amplitudes for these graphs is

$$
\begin{aligned}
A_{2}(l)= & -\frac{v_{1}^{2} \Lambda^{2 \alpha+2-2 l} \int d x \Delta^{2 l}(x)}{[(\alpha+1-l) !]^{2} 2^{2 \alpha+3-2 l}(2 l) !}+\frac{v_{1} v_{3} g \Lambda^{2 \alpha+2-2 l} \int d x \Delta^{2 l}(x)}{(\alpha+1-l) !(\alpha-l) ! 2^{2 \alpha+1-2 l}(2 l) !} \\
& -\frac{v_{3}^{2} g^{2} \Lambda^{2 \alpha+2-2 l} \int d x \Delta^{2 l}(x)}{[(\alpha-l) !]^{2} 2^{2 \alpha+1-2 l}(2 l) !}+\frac{v_{3}^{2} \Lambda^{2 \alpha-2 l} \int d x \Delta^{2 l}(x) \operatorname{Tr} \bar{S}(x) S(x)}{[(\alpha-l) !]^{22} 2^{2 \alpha+2-2 l}(2 l) !} .
\end{aligned}
$$

Note that we must sum over all possible values of $l$. In all of the above terms except the last, $l$ ranges from 1 to $\infty$, but in the second $v_{3}^{2}$ term $l$ ranges from 0 to $\infty$. To simplify the Feynman integral in this term representing the boson- and fermion-exchange graph, we use the following identity stated in [9]:

$$
\int d x \Delta^{l}(x) \operatorname{Tr} \bar{S}(x) S(x)=\frac{2 g^{2}(l+2)}{l+1} \int d x[\Delta(x)]^{l+2}-\frac{2}{l+1} \Lambda^{l+1}, \quad l \geq 0,
$$

where $S(x)$ and $\Delta(x)$ are the fermion and boson propagators in coordinate space: 


$$
\begin{aligned}
S(x) & =(g-i \not \partial) \Delta(x), \\
\Delta(x) & =\frac{1}{2 \pi} K_{0}(g|x|) .
\end{aligned}
$$

When we simplify the expression $A_{2}(l)$ there is a remarkable cancellation that occurs; all integrals over $\Delta^{2 l}(x)$ cancel independently of the value of $\alpha$ and we obtain the following simple sum:

$$
A_{2}=\sum_{l} A_{2}(l)=-g^{2} \delta^{2} \Lambda^{2 \alpha+1}[(2 \alpha+1) !]^{2} 2^{-2 \alpha-5} \sum_{l=1}^{\infty} \frac{2^{2 l}}{(2 l-1) ![(\alpha+1-l) !]^{2}} .
$$

Third, we consider the two-vertex graphs constructed from $v_{2}$ and $v_{4}$, which are shown in Fig. 4(c). The sum of the amplitudes for these graphs is identical to $A_{2}$ in Eq. (4.9) with the replacement $\alpha \rightarrow \beta$ :

$$
A_{3}=\left.A_{2}\right|_{\alpha \rightarrow \beta} .
$$

Fourth, we examine the two-vertex graphs constructed from one of $v_{1}$ and $v_{3}$ and one of $v_{2}$ and $v_{4}$. These graphs are shown in Fig. 4(d). For each value of $l$ the sum of the amplitudes for these graphs is

$$
\begin{aligned}
A_{4}(l)= & -\frac{v_{1} v_{2} \Lambda^{\alpha+\beta+2-2 l} \int d x \Delta^{2 l}(x)}{(\alpha+1-l) !(\beta+1-l) ! 2^{\alpha+\beta+2-2 l}(2 l) !}+\frac{v_{1} v_{4} g \Lambda^{\alpha+\beta+2-2 l} \int d x \Delta^{2 l}(x)}{(\alpha+1-l) !(\beta-l) ! 2^{\alpha+\beta+1-2 l}(2 l) !} \\
& +\frac{v_{2} v_{3} g \Lambda^{\alpha+\beta+2-2 l} \int d x \Delta^{2 l}(x)}{(\alpha-l) !(\beta+1-l) ! 2^{\alpha+\beta+1-2 l}(2 l) !}-\frac{v_{3} v_{4} g^{2} \Lambda^{\alpha+\beta+2-2 l} \int d x \Delta^{2 l}(x)}{(\alpha-l) !(\beta-l) ! 2^{\alpha+\beta-2 l}(2 l) !} \\
& +\frac{v_{3} v_{4} \Lambda^{\alpha+\beta-2 l} \int d x \Delta^{2 l}(x) \operatorname{Tr} \bar{S}(x) S(x)}{(\alpha-l) !(\beta-l) ! 2^{\alpha+\beta+1-2 l}(2 l) !} .
\end{aligned}
$$

Again, we must sum over all possible values of $l$; in all of the above terms except the last, $l$ ranges from 1 to $\infty$, but in the $v_{3} v_{4}$ term representing boson- and fermion-exchange, $l$ ranges from 0 to $\infty$. Also, we again use the integral identity in Eq. (4.7). As before, when we simplify the expression $A_{4}(l)$ there is a remarkable cancellation of the $\Delta^{2 l}(x)$ integrals for all values of $\alpha$ and $\beta$. We obtain the following simple sum:

$$
A_{4}=\sum_{l} A_{4}(l)=g^{2} \delta^{2} \Lambda^{\alpha+\beta+1}(2 \alpha+1) !(2 \beta+1) ! \sum_{l=1}^{\infty} \frac{2^{2 l-\alpha-\beta-4}}{(2 l-1) !(\alpha+1-l) !(\beta+1-l) !} .
$$

Next, we consider the two-vertex graphs constructed from $v_{5}$ and $v_{7}$, which are shown in Fig. 4(e). For each value of $l$ the sum of the amplitudes for these graphs is

$$
\begin{aligned}
A_{5}(l)= & -\frac{v_{5}^{2} \Lambda^{2 \alpha+2 \gamma-2 l} \int d x \Delta^{2 l+1}(x)}{[(\alpha+\gamma-l) !]^{2} 2^{2 \alpha+2 \gamma+1-2 l}(2 l+1) !} \\
& +\frac{v_{5} v_{7} g \Lambda^{2 \alpha+2 \gamma-2 l} \int d x \Delta^{2 l+1}(x)}{(\alpha+\gamma-l) !(\alpha+\gamma-1-l) ! 2^{2 \alpha+2 \gamma-1-2 l}(2 l+1) !} \\
& -\frac{v_{7}^{2} g^{2} \Lambda^{2 \alpha+2 \gamma-2 l} \int d x \Delta^{2 l+1}(x)}{[(\alpha+\gamma-1-l) !]^{2} 2^{2 \alpha+2 \gamma-1-2 l}(2 l+1) !} \\
& +\frac{v_{7}^{2} \Lambda^{2 \alpha+2 \gamma-2-2 l} \int d x \Delta^{2 l+1}(x) \operatorname{Tr} \bar{S}(x) S(x)}{[(\alpha+\gamma-1-l) !]^{2} 2^{2 \alpha+2 \gamma-2 l}(2 l+1) !} .
\end{aligned}
$$


Again, we must sum over all possible values of $l$; in all of the above terms $l$ ranges from 0 to $\infty$. Also, in the boson- and fermion-exchange graph in the last term, we again use the integral identity in Eq. (4.7). When we simplify the expression $A_{5}(l)$ there is no longer any cancellation for arbitrary values of $\alpha$. Thus, the final result still contains an integral over $\Delta^{2 l+1}(x)$. We obtain the following sum:

$$
\begin{aligned}
A_{5}=\sum_{l} A_{5}(l)= & \pi^{2} g^{2} \delta^{2} \alpha^{2} \Lambda^{2 \alpha+2 \gamma}[(2 \alpha+2 \gamma-1) !]^{2} 2^{-2 \alpha-2 \gamma-3}\left\{\sum_{l=0}^{\infty} \frac{2^{2 l}}{(2 l) ![(\alpha+\gamma-l) !]^{2}}\right. \\
& \left.+\sum_{l=0}^{\infty} \frac{g^{2} 2^{2 l}\left[(2 \alpha+2 \gamma)^{2}-1\right]\left[(2 \alpha+2 \gamma)^{2}+4 l+1\right] \int d x \Delta^{2 l+1}(x)}{(2 l+1) ![(\alpha+\gamma-l) !]^{2} \Lambda^{2 l}}\right\} .
\end{aligned}
$$

Sixth, we consider the two-vertex graphs constructed from $v_{6}$ and $v_{8}$, which are shown in Fig. 4(f). The sum of the amplitudes for these graphs is identical to $A_{5}$ in Eq. (4.14) with the replacement $\alpha \rightarrow \beta$ :

$$
A_{6}=\left.A_{5}\right|_{\alpha \rightarrow \beta} .
$$

The seventh and last class of graphs is constructed from one of $v_{5}$ and $v_{7}$ and one of $v_{6}$ and $v_{8}$. These graphs are shown in Fig. $4(\mathrm{~g})$. It is easy to see that the amplitudes for all such graphs are proportional to $\alpha \beta$. Thus, anticipating that at the end of the calculation we will set both $\alpha=0$ and $\beta=0$, we need not calculate the amplitude $A_{7}$ because it will not contribute to the ground-state energy density. (Note that before we set $\alpha$ and $\beta$ to 0 , we must apply the differential operator $\mathcal{D}$ in Eq. (2.12). This operator does not have a mixed derivative term and thus it cannot eliminate both factors of $\alpha$ and $\beta$.)

The final part of this calculation consists of applying the operator $\mathcal{D}$ in Eq. (2.12) to $A_{1}+A_{2}+A_{3}+A_{4}+A_{5}+A_{6}+A_{7}$ and setting $\alpha=0, \beta=0$, and $\gamma=\frac{1}{2}$. After a rather lengthy calculation, we obtain the result

$$
\begin{aligned}
& E_{\text {ground state }}=\left.\mathcal{D} \sum_{j=1}^{7} A_{j}\right|_{\alpha=\beta=0, \gamma=1 / 2} \\
= & \frac{1}{16} \delta^{2} g^{2} \Lambda\left[2 \psi^{\prime}\left(\frac{3}{2}\right)-2 \pi^{2}+\sum_{l=0}^{\infty} \frac{\sqrt{\pi} \Gamma\left(l-\frac{1}{2}\right)}{\left(l-\frac{1}{2}\right) \Gamma(l+1)}-\sum_{l=1}^{\infty} \frac{\sqrt{\pi} \Gamma(l)}{l \Gamma\left(l+\frac{3}{2}\right)}\right]+\mathrm{O}\left(\delta^{3}\right) .
\end{aligned}
$$

The sums in this expression may be evaluated easily and we obtain the result

$$
E_{\text {ground state }}=0+\mathrm{O}\left(\delta^{3}\right) .
$$

\section{B. Calculation of $\langle\phi\rangle$ to second order in $\delta$}

The graphs that contribute to the vacuum expectation value of the scalar field arise either from one-vertex graphs constructed from the odd vertices, $v_{5}$ through $v_{8}$, or from two vertex graphs with one odd vertex and one even vertex. Some of these graphs are shown in Fig. 5. Again, they fall into natural classes. We first consider the five two-vertex graphs in 
which an even number of bosons are exchanged between the pairs of $\alpha$ vertices $\left(v_{1}, v_{3}\right)$ and $\left(v_{5}, v_{7}\right)$. For a given $l$ the sum of the five amplitudes is

$$
\begin{aligned}
& \frac{v_{1} v_{5} \Lambda^{2 \alpha+2 \gamma+1-2 l} \int d x \Delta^{2 l}}{(\alpha+1-l) !(\alpha+\gamma-l) !(2 l) ! 2^{2 \alpha+\gamma+1-2 l}}-\frac{v_{1} v_{7} g \Lambda^{2 \alpha+2 \gamma+1-2 l} \int d x \Delta^{2 l}}{(\alpha+1-l) !(\alpha+\gamma-1-l) !(2 l) ! 2^{2 \alpha+\gamma-2 l}} \\
& -\frac{v_{3} v_{5} g \Lambda^{2 \alpha+2 \gamma+1-2 l} \int d x \Delta^{2 l}}{(\alpha-l) !(\alpha+\gamma-l) !(2 l) ! 2^{2 \alpha+\gamma-2 l}}+\frac{v_{3} v_{7} g^{2} \Lambda^{2 \alpha+2 \gamma+1-2 l} \int d x \Delta^{2 l}}{(\alpha-l) !(\alpha+\gamma-1-l) !(2 l) ! 2^{2 \alpha+\gamma-1-2 l}} \\
& -\frac{v_{3} v_{7} \Lambda^{2 \alpha+2 \gamma-1-2 l} \int d x \Delta^{2 l} \operatorname{Tr} \bar{S}(x) S(x)}{(\alpha-l) !(\alpha+\gamma-1-l) !(2 l) ! 2^{2 \alpha+\gamma-2 l}} .
\end{aligned}
$$

There are five more graphs in which an odd number of bosons are exchanged. The sum of the amplitudes of these graphs is similar to the result in Eq. (4.18) and we do not give it here. Furthermore, there are ten corresponding graphs in which $\alpha$ is replaced by $\beta$ and these are constructed from the vertices $v_{2}, v_{4}, v_{6}$, and $v_{8}$. When these twenty amplitudes are combined and summed over $l$ no dramatic cancellation like that in the calculation of the ground-state energy density occurs. Thus, we are left with an infinite sum over integrals of the coordinate space propagator $\Delta(x)$.

Next, we consider the contributions of the twenty graphs, analogous to those above, that are constructed from one $\alpha$ vertex and one $\beta$ vertex. That is, we construct all possible multi-boson exchange graphs from the vertices $\left(v_{1}, v_{3}\right)$ connected to $\left(v_{6}, v_{8}\right)$, and $\left(v_{2}, v_{4}\right)$ connected to $\left(v_{5}, v_{7}\right)$. This calculation simplifies dramatically because only one- and twoparticle exchange graphs survive when we apply the derivative operator in Eq. (2.12) and set $\alpha=\beta=0$ and $\gamma=1 / 2$.

Last, we include the four single-vertex graphs constructed from the vertices $v_{5}, v_{6}, v_{7}$, and $v_{8}$.

When we combine all of these calculations we obtain the following result:

$$
\begin{aligned}
& \langle\phi\rangle=i \sqrt{\pi \Lambda / 2}\left\{-\delta+\delta^{2}[1-\ln 2\right. \\
& \left.\left.+g^{2} \int d x \Delta(x)\left(\frac{\Delta(x)}{2 \Lambda}\left[\ln \left(\frac{\Lambda}{2}\right)+\psi(1)\right]+\left[1+\frac{\Delta(x)}{\Lambda}\right] \ln \left[1+\frac{\Delta(x)}{\Lambda}\right]\right)\right]+\mathrm{O}\left(\delta^{3}\right)\right\} .
\end{aligned}
$$

Note that any positive integer power of the propagator $\Delta(x)$ in Eq. (4.8) is integrable. However, the value of the propagator at the origin $\Lambda=\Delta(0)$ is a divergent quantity. Therefore, the function $\Delta(x) / \Lambda$ vanishes everywhere except at $x=0$, where it is unity. Hence, the integral involving this ratio in Eq. (4.19) exists and vanishes. Thus, our final result for the one-point function, which measures the parity symmetry breaking in this theory, is

$$
\langle\phi\rangle=i \sqrt{\pi \Lambda / 2}\left[-\delta+\delta^{2}(1-\ln 2)+\mathrm{O}\left(\delta^{3}\right)\right] .
$$

The fact that the theory is supersymmetric reduces the degree of divergence of this result. At intermediate stages of the calculation, the coefficient of $\delta^{2}$ is proportional to $\Lambda^{1 / 2} \ln \Lambda$. However, when the boson and fermion contributions are combined, all terms containing $\ln \Lambda$ cancel exactly. Thus, the higher-order result is no more divergent than the leading-order result. 


\section{Calculation of the fermion-boson mass ratio to second order in $\delta$}

We do not discuss this calculation in detail here because a similar one is explained in Ref. [10]. The calculation done here is more elaborate because there are twice as many vertices but the necessary calculations are routine. Our result is

$$
R=\frac{M_{\text {fermion }}}{M_{\text {boson }}}=1+\mathrm{O}\left(\delta^{3}\right),
$$

which is consistent with the theory being supersymmetric.

\section{CONCLUSIONS}

In the Schwinger model of two-dimensional electrodynamics with massless fermions there is an anomaly. If the one-fermion-loop contribution to the photon propagator is calculated naively one obtains a product of two factors; the first factor vanishes in two-dimensional space, and the second factor is a divergent integral. If one is not careful, one gets a quantity that is formally 0 . However, because the integral is divergent, one must evaluate this product by introducing a regulator; dimensional regulation is an acceptable procedure. As the regulator is removed one obtains a finite, nonvanishing result for the anomaly, namely, the famous number $e^{2} / \pi$. In general, one looks for an anomaly when there is a naive cancellation involving divergent quantities that must be regulated. The question that is raised in this paper is, do we have an anomaly in the $\delta$ expansion that breaks supersymmetry? Specifically, is there an anomaly associated with the cancellation that gives a vanishing ground-state energy density in Eq. (3.7)?

\section{A. Dimensional regularization of the ground-state energy density}

In the derivation of Eq. (3.7) we combine two numbers that are divergent to obtain 0 . There are several ways to regulate the integral representing $\Lambda$. For example, if we use dimensional regulation and evaluate $\Lambda$ in $2-\epsilon$ dimensions, then for small positive $\epsilon$

$$
\begin{aligned}
\Lambda & \sim \frac{1}{2 \pi} \int_{0}^{\infty} d p \frac{p^{1-\epsilon}}{p^{2}+g^{2}} \quad(\epsilon \rightarrow 0+) \\
& =\frac{1}{4 \pi} g^{-\epsilon} \int_{0}^{1} d u u^{-1+\epsilon / 2}(1-u)^{-\epsilon / 2} \\
& =\frac{1}{4 \pi} g^{-\epsilon} \Gamma\left(\frac{\epsilon}{2}\right) \Gamma\left(1-\frac{\epsilon}{2}\right) \\
& =\frac{1}{4} g^{-\epsilon} \frac{1}{\sin (\pi \epsilon / 2)} \sim \frac{1}{2 \pi \epsilon} g^{-\epsilon} \quad(\epsilon \rightarrow 0+) .
\end{aligned}
$$

Furthermore, in $d$-dimensional space, the representation of the Dirac matrices has rank $2^{d / 2}$. Thus, the trace of a unit matrix in $2-\epsilon$ dimensions is

$$
\operatorname{Tr} \mathbb{1}=2^{(2-\epsilon) / 2} \sim 2-\epsilon \ln 2 \quad(\epsilon \rightarrow 0+) .
$$


Thus, the coefficient of the second graph amplitude in Eq. (3.6) should be multiplied by $1-\frac{1}{2} \epsilon \ln 2$.

We now see that the two graphs do not exactly cancel; rather, the difference in the numerical coefficients is of order $\epsilon$. Combining the two graphs in (3.6) now gives

$$
\begin{aligned}
\epsilon(\ln 2) \delta g^{2} \frac{(2 \alpha+1) ! \Lambda^{\alpha+1}}{2^{\alpha+2} \alpha !} & =\frac{\epsilon \ln 2}{2 \sqrt{\pi}} \delta g^{2} 2^{\alpha} \Gamma\left(\alpha+\frac{3}{2}\right) \Lambda^{\alpha+1} \\
& \sim \frac{\ln 2}{4 \pi^{3 / 2}} \delta g^{2} \Gamma\left(\alpha+\frac{3}{2}\right)(2 \Lambda)^{\alpha} \quad(\epsilon \rightarrow 0+) .
\end{aligned}
$$

If we now differentiate with respect to $\alpha$ and set $\alpha=0$, we obtain for the ground-state energy density

$$
\begin{aligned}
E_{\text {ground state }} & =\frac{\ln 2}{8 \pi} \delta g^{2}\left[\psi\left(\frac{3}{2}\right)+\ln (2 \Lambda)\right]+\mathrm{O}\left(\delta^{2}\right) \\
& \sim \frac{\ln 2}{8 \pi} \delta g^{2}\left[\psi\left(\frac{3}{2}\right)-\ln (\pi \epsilon)\right]+\mathrm{O}\left(\delta^{2}\right) \quad(\epsilon \rightarrow 0+) .
\end{aligned}
$$

Because this is a positive number, it suggests that supersymmetry may be broken.

Of course, dimensional regulation violates supersymmetry. Thus, it is not clear whether the nonzero result in Eq. (5.4) is correct or is merely an artifact of the regularization scheme being used.

\section{B. Proper-time regularization}

If a supersymmetric regulation exists then, of course, there will be no anomaly. Conversely, if we could establish rigorously that there does not exist any supersymmetric regulation of the delta expansion, then there really would be a breaking of supersymmetry. We do not know for certain whether a supersymmetric regulation of the $\delta$ expansion exists. However, we believe that we have found a relatively simple way to regulate the $\delta$ expansion that is consistent with supersymmetry and thus we believe that there is no anomalous structure and that the ground-state energy is truly identically zero. This suggests that while it is relatively easy to break parity symmetry, supersymmetry is extremely rigid and is very difficult to break.

Our regulation scheme, which we believe respects supersymmetry invariance, is a variant of the proper-time method due to J. Schwinger [15]. (It is well known that the propertime method correctly yields the anomaly in the Schwinger model.) Here, we will use this method to define, in an invariant way, the divergent integral $\Lambda$. To make contact with the dimensionally regulated result above, let us work in $d$ dimensions:

$$
\Lambda=\int \frac{d^{d} p}{(2 \pi)^{d}} \frac{1}{p^{2}+g^{2}}=\int \frac{d^{d} p}{(2 \pi)^{2}} \int_{s_{0}}^{\infty} d s e^{-s\left(p^{2}+g^{2}\right)} .
$$

Here, $s_{0}$ is a proper-time regulator to be taken to 0 at the end of the calculation. We now interchange the order of integration in (5.5), and express the momentum integral as the product of $d$ one-dimensional integrals: 


$$
\int_{-\infty}^{\infty} \frac{d p}{2 \pi} e^{-s p^{2}}=\frac{1}{2 \sqrt{\pi s}} .
$$

Then, the integral representing $\Lambda$ is immediately expressed in terms of a single regulated integral:

$$
\Lambda=\int_{s_{0}}^{\infty} d s e^{-s g^{2}}\left(\frac{1}{2 \sqrt{\pi s}}\right)^{d}=\frac{g^{d-2}}{2^{d} \pi^{d / 2}} \int_{g^{2} s_{0}}^{\infty} d x x^{-d / 2} e^{-x} .
$$

If we set $d=2-\epsilon$ as in the previous subsection, the integral converges when $s_{0}=0$, and we obtain the same result as in (5.1),

$$
\Lambda \sim \frac{1}{4 \pi} \Gamma\left(\frac{\epsilon}{2}\right) .
$$

However, if we want to preserve supersymmetry, we must remain in two dimensions, in which case the integral depends logarithmically upon $s_{0}$ :

$$
d=2: \quad \Lambda=\frac{1}{4 \pi} \int_{g s_{0}}^{\infty} \frac{d x}{x} e^{-x} \sim-\frac{1}{4 \pi}\left[\gamma+\ln \left(g s_{0}\right)\right],
$$

where $\gamma$ is Euler's constant.

The obvious advantage of this regulation scheme is that it treats bosons and fermions on an equal footing; the renormalization of the boson and fermion masses is identical. With this

regulation scheme, the ground-state energy density is zero, as expected. Thus, we believe that there is no anomaly in the $\delta$ expansion.

\section{ACKNOWLEDGEMENT}

We thank A. Das, L. Gamberg, and M. Grisaru for illuminating discussions, and we are grateful to the U.S. Department of Energy for financial support. 


\section{REFERENCES}

[1] C. M. Bender, K. A. Milton, M. Moshe, S. S. Pinsky, and L. M. Simmons, Jr., Phys. Rev. Lett. 58, 2615 (1987); C. M. Bender, K. A. Milton, M. Moshe, S. S. Pinsky, and L. M. Simmons, Jr., Phys. Rev. D 37, 1472 (1988).

[2] C. M. Bender, K. A. Milton, S. S. Pinsky, and L. M. Simmons, Jr., J. Math. Phys. 30, 1447 (1989).

[3] C. M. Bender, S. Boettcher, and K. A. Milton, J. Math. Phys. 32, 3031 (1991); B. Abraham-Shrauner, C. M. Bender, and R. N. Zitter, J. Math. Phys. 33, 1335 (1992).

[4] C. M. Bender and H. F. Jones, Phys. Rev. D 38, 2526 (1988); H. T. Cho, K. A. Milton, J. Cline, S. S. Pinsky, and L. M. Simmons, Jr., Nucl. Phys. B 329, 574 (1990).

[5] C. M. Bender, F. Cooper, and K. A. Milton, Phys. Rev. D 40, 1354 (1989); C. M. Bender, S. Boettcher, and K. A. Milton, Phys. Rev. D 45, 639 (1992); C. M. Bender, F. Cooper, K. Milton, M. Moshe, S. S. Pinsky, and L. M. Simmons, Jr., Phys. Rev. D 45, 1248 (1992); C. M. Bender, K. A. Milton, and M. Moshe, Phys. Rev. D 45, 1261 (1992).

[6] C. M. Bender, F. Cooper, and K. A. Milton, Phys. Rev. D 39, 3684 (1989); C. M. Bender, F. Cooper, G. Kilkup, P. Roy, and L. M. Simmons, Jr., J. Stat. Phys. 64, 395 (1991).

[7] C. M. Bender and T. Rebhan, Phys. Rev. D 41, 3269 (1990).

[8] C. M. Bender, K. A. Milton, S. S. Pinsky, and L. M. Simmons, Jr., J. Math. Phys. 31, $2722(1990)$.

[9] C. M. Bender, K. A. Milton, S. S. Pinsky, and L. M. Simmons, Jr., Phys. Lett. B 205, 493 (1988).

[10] C. M. Bender and K. A. Milton, Phys. Rev. D 38, 1310 (1988).

[11] C. M. Bender and K. A. Milton, Phys. Rev. D 55, R3255 (1997).

[12] D. Bessis, private communication.

[13] L. Alvarez-Gaumé, D. Z. Freedman, and M. T. Grisaru, Harvard University report No. HUTHP 81/B111 (1981), unpublished; T. Murphy and L. O'Raifeartaigh, Nucl. Phys. B218, 484 (1983); E. Witten, Nucl. Phys. 188, 513 (1981).

[14] C. M. Bender and A. Turbiner, Phys. Lett. A 173, 442 (1993).

[15] J. Schwinger, Phys. Rev. 82, 664 (1951). 


\section{FIGURES}

FIG. 1. The two graphs contributing to the ground-state energy to first order in $\delta$. These graphs are constructed from vertices having an even number of boson lines. The symmetry numbers are shown beside each graph.

FIG. 2. The two graphs contributing to the one-point Green's function to first order in $\delta$. These graphs are constructed from vertices having an odd number of boson lines. The symmetry numbers are shown beside each graph.

FIG. 3. One-particle-irreducible graphs contributing to the mass renormalization of the fermion and the boson to order $\delta$. There is one graph for the fermion mass shift and two graphs for the boson mass shift. All graphs are constructed from vertices having an even number of boson lines. The symmetry numbers for the graphs are shown beside each graph.

FIG. 4. The thirty graphs contributing to the ground-state energy to second order in $\delta$. These graphs are constructed from the eight vertices in Eq. (4.3). We have organized the graphs into sets for which the amplitudes combine in a natural way. These sets are labeled (a)-(g).

FIG. 5. Five of the graphs contributing to $\langle\phi\rangle$ to second order in $\delta$. These graphs are constructed from the eight vertices in Eq. (4.3). 

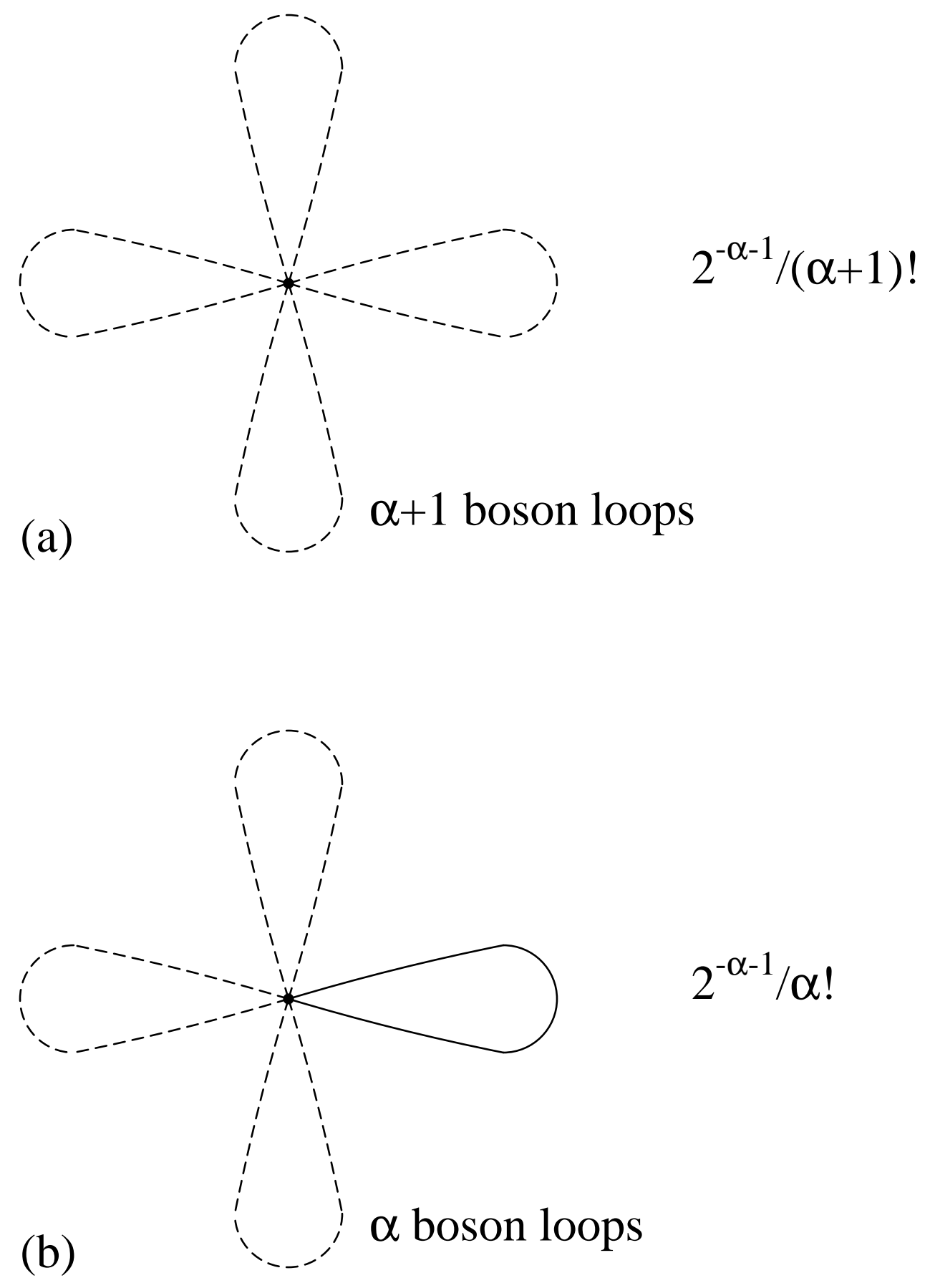

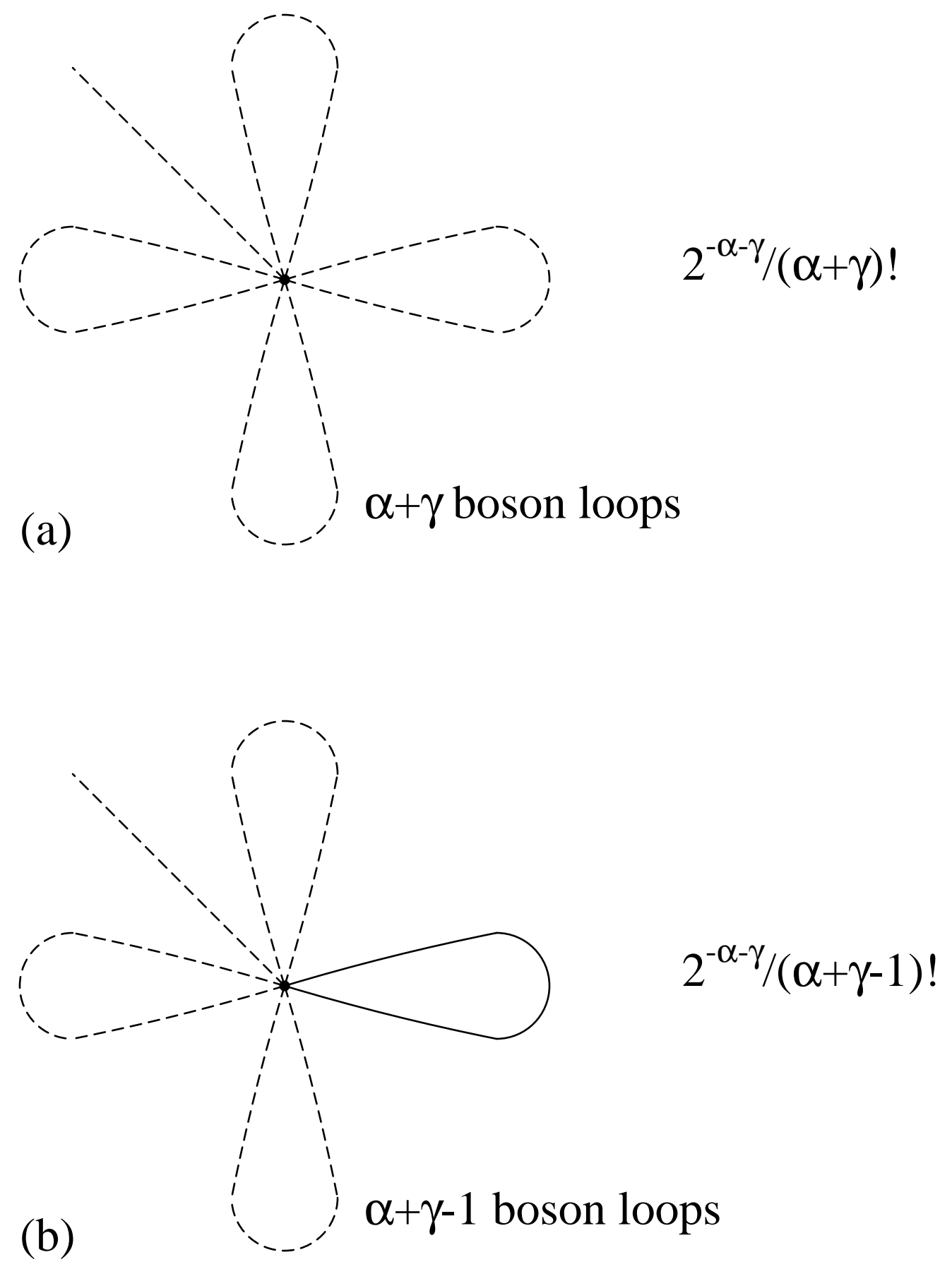


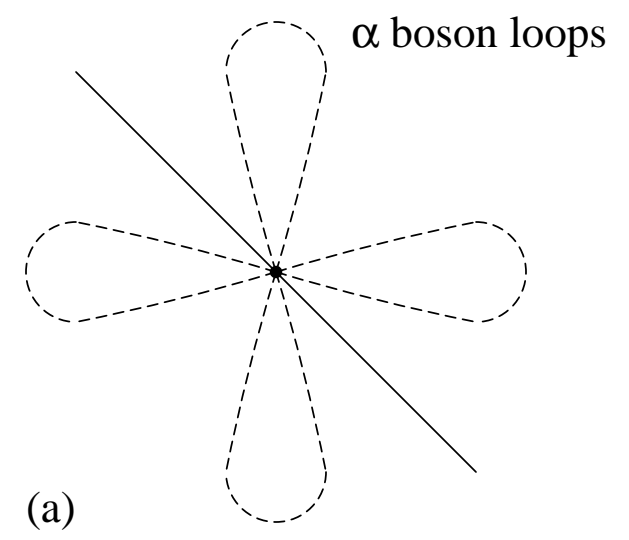

$$
2^{-\alpha} / \alpha !
$$

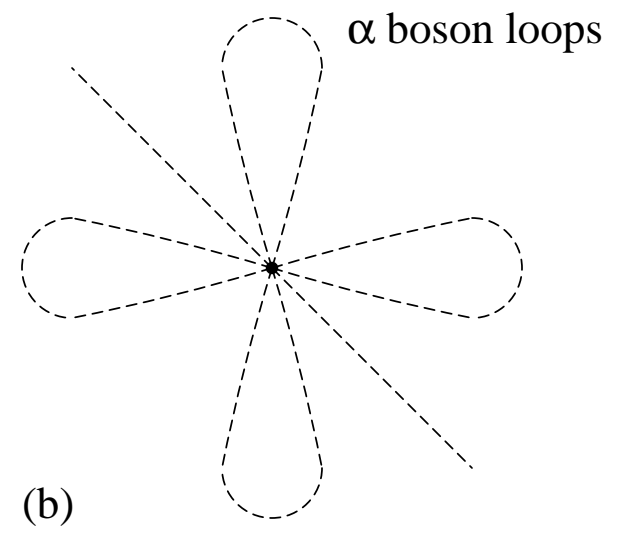

$2^{-\alpha} / \alpha !$

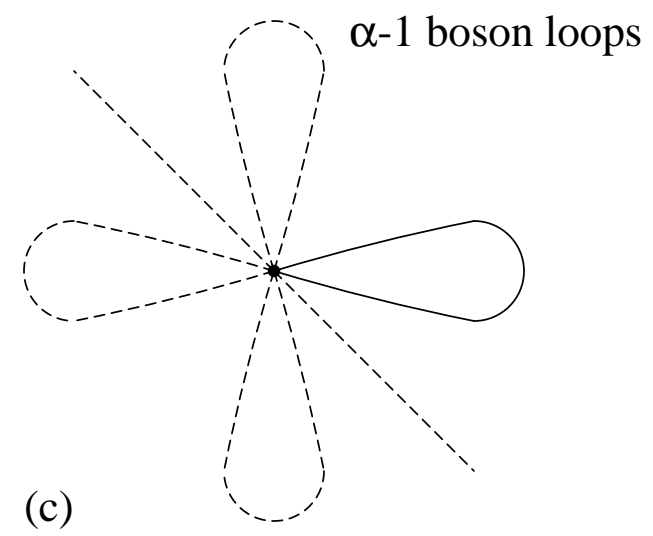



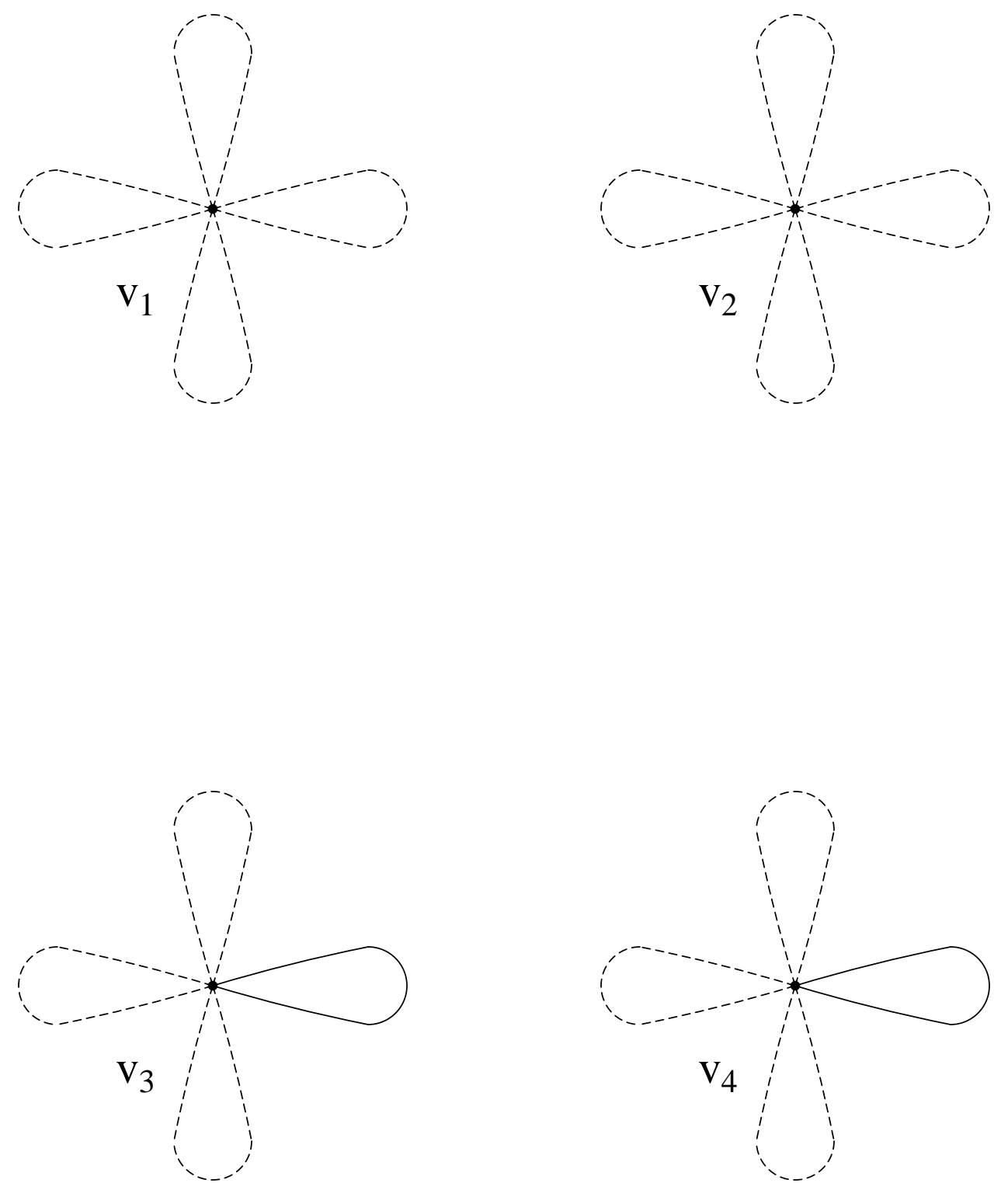

(a) 

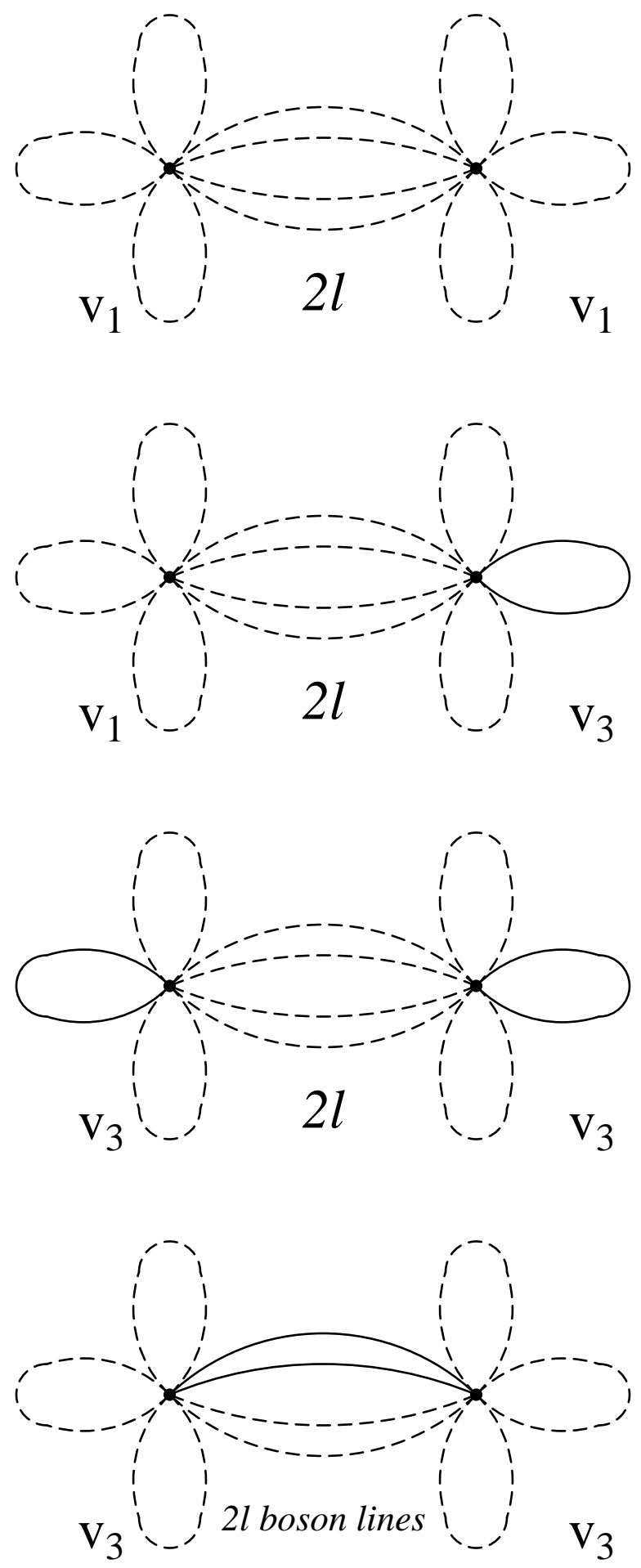

(b) 

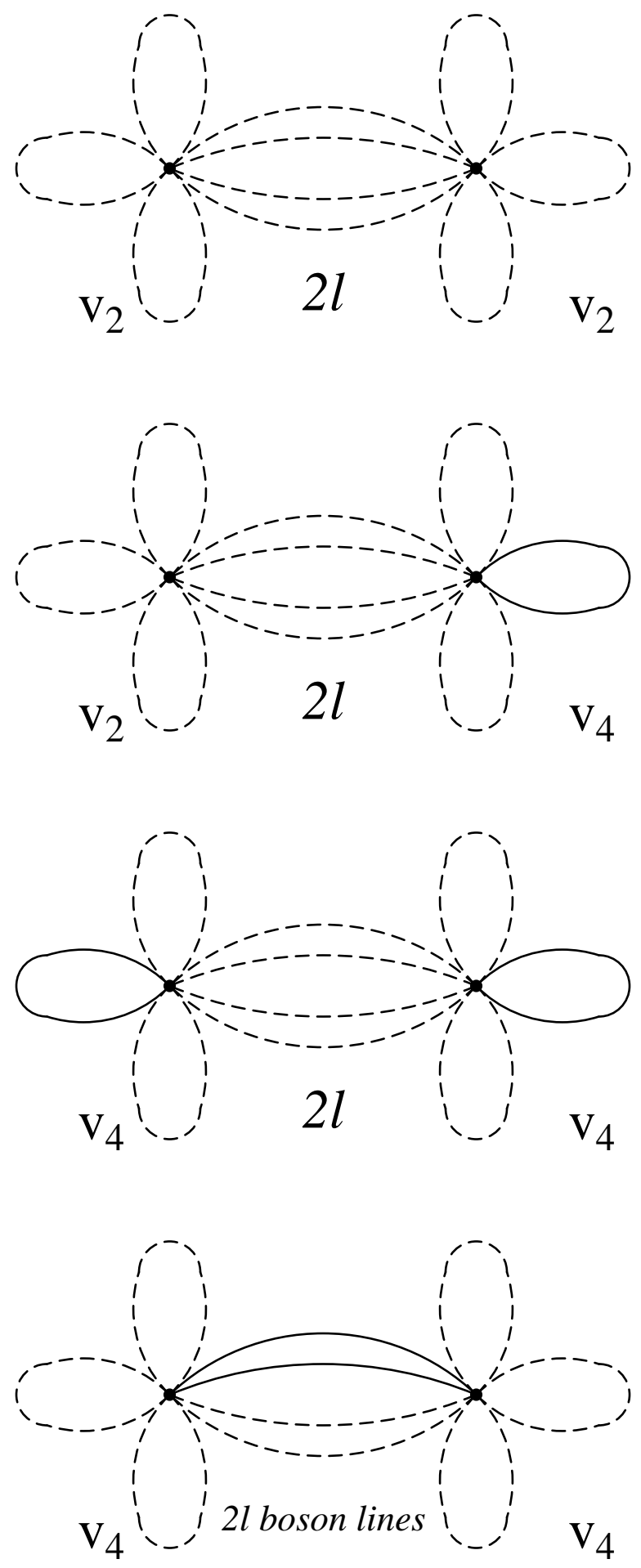

(c) 

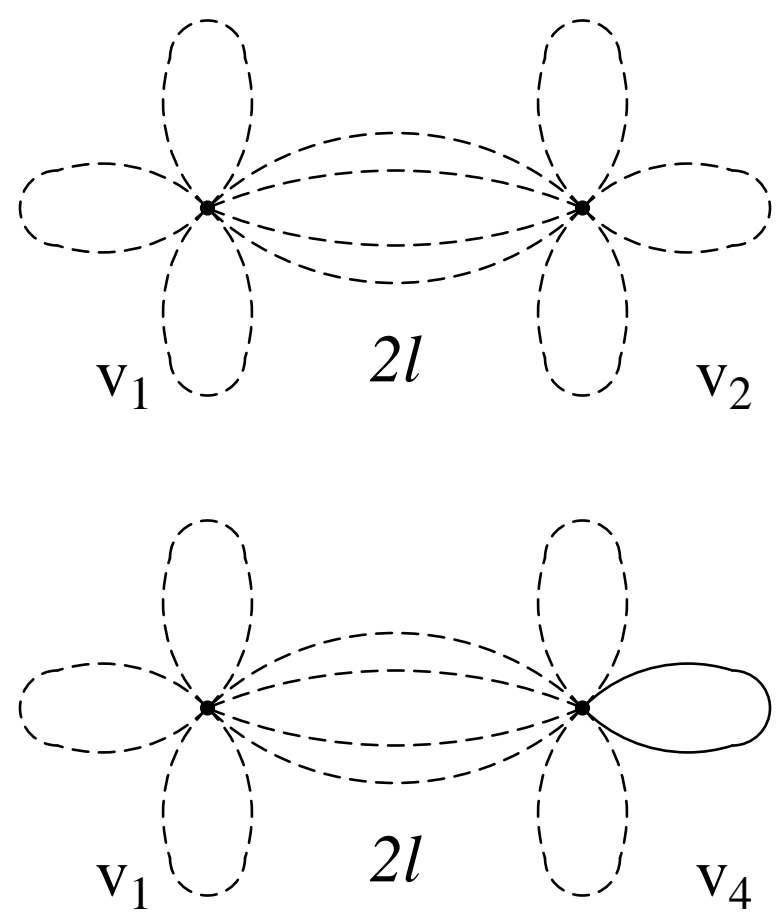

(d)
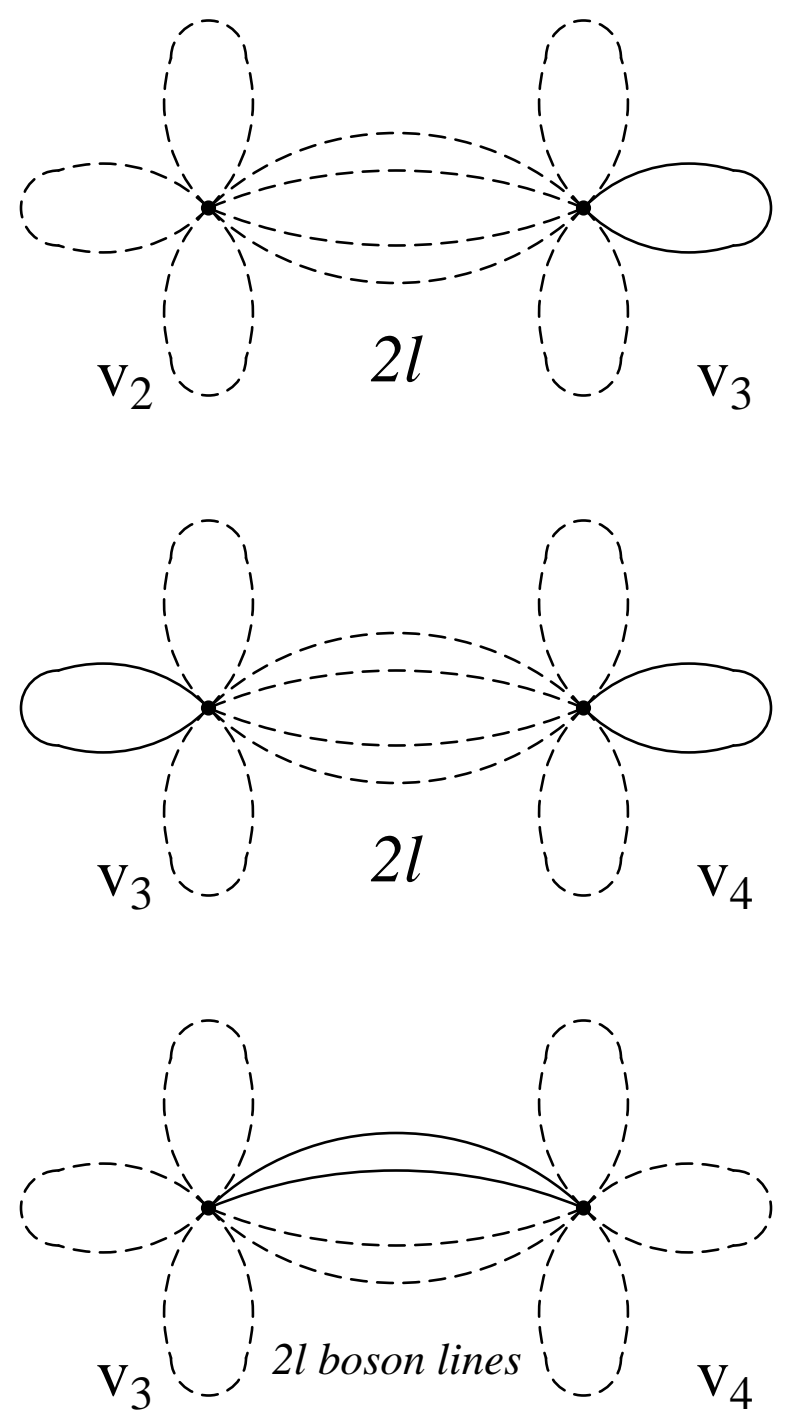

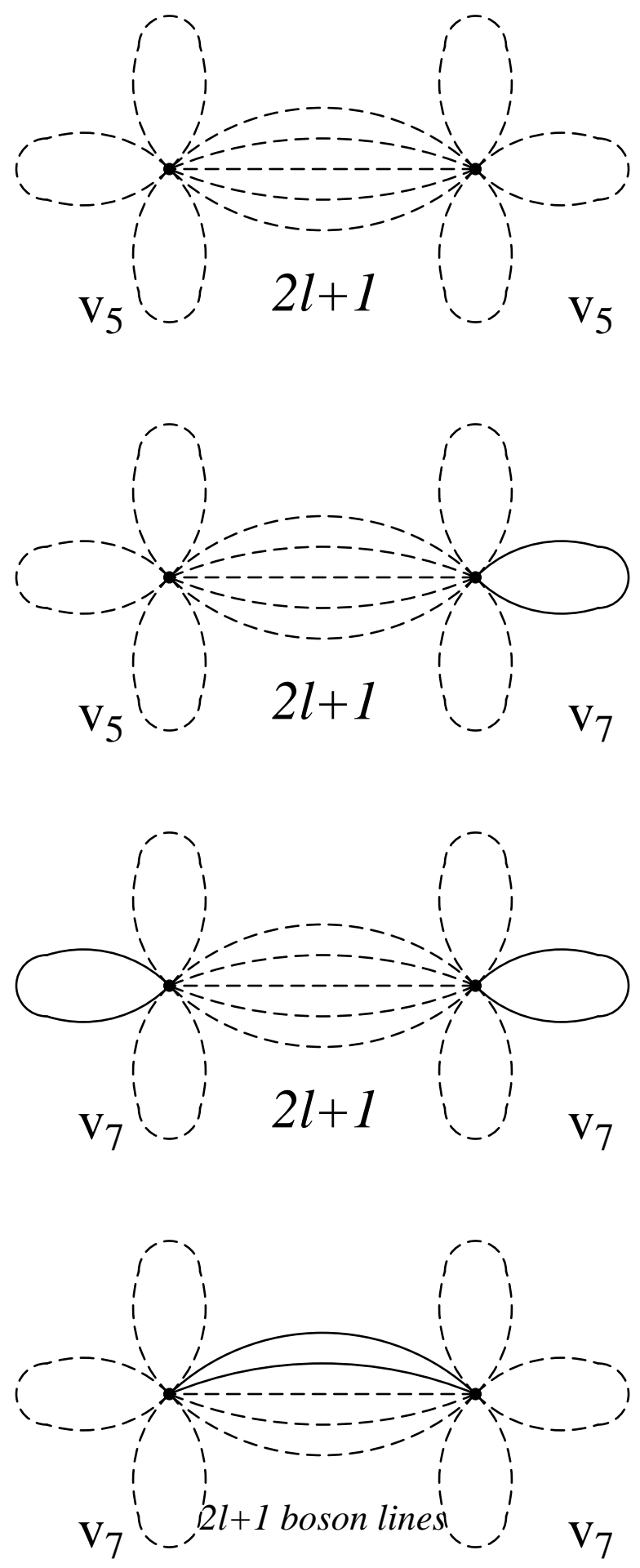

(e) 

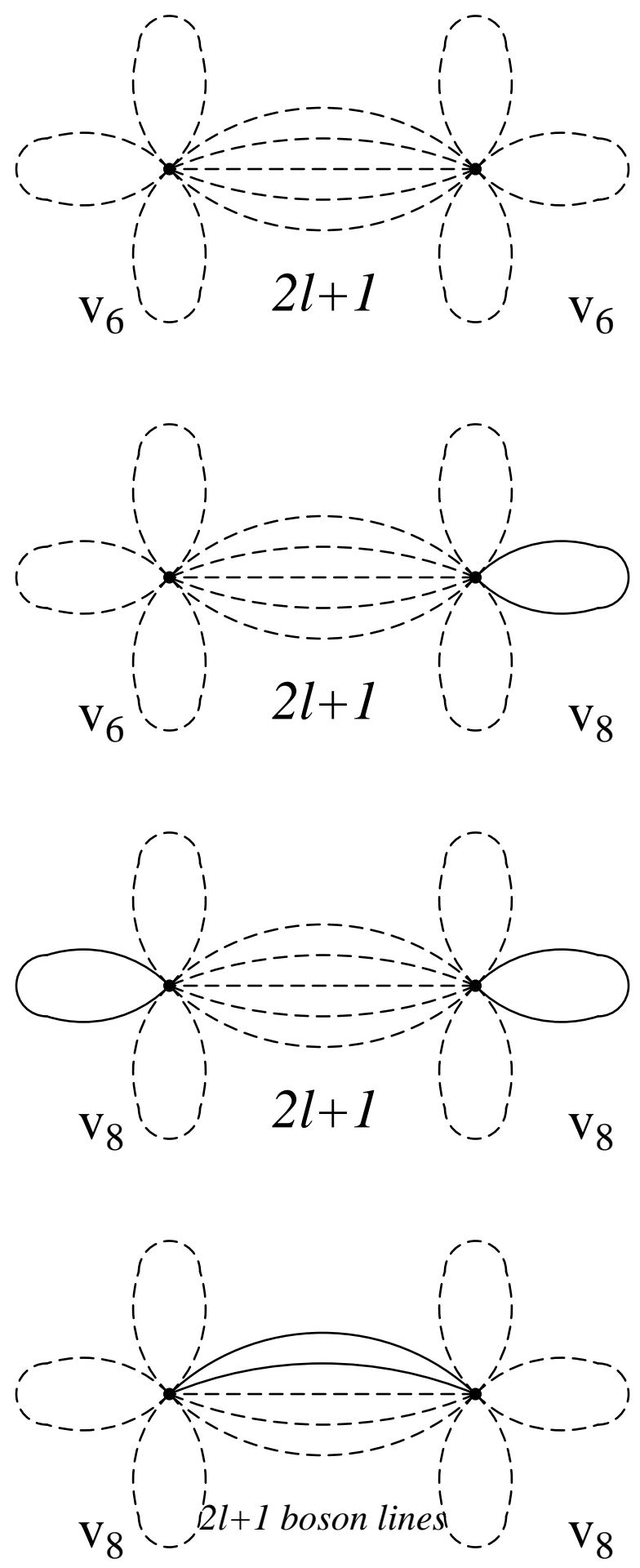

(f) 

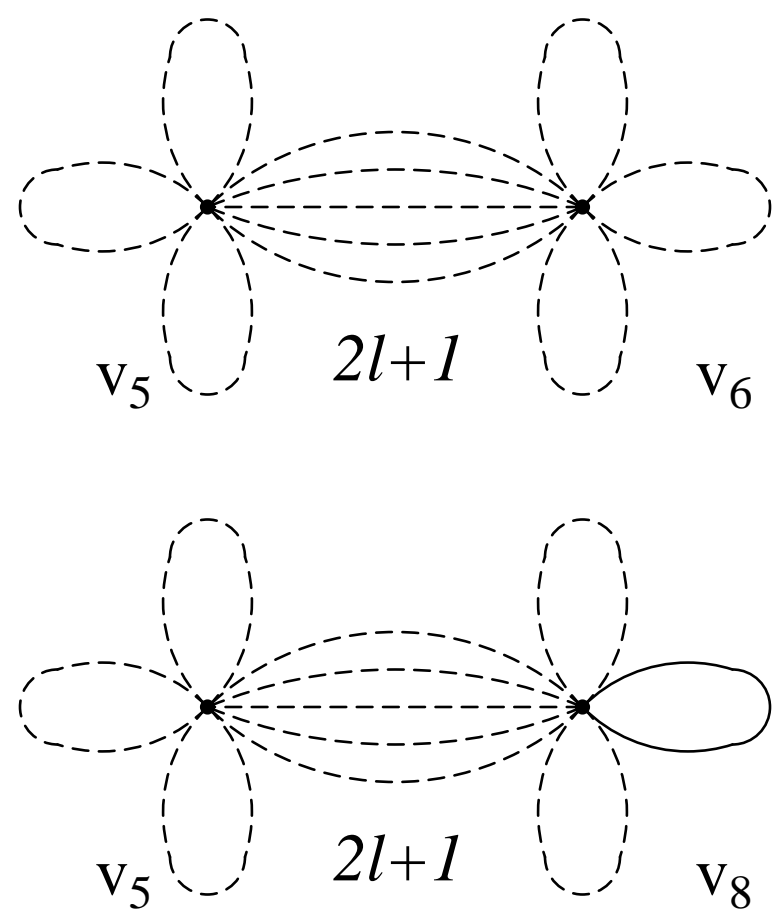

(g)
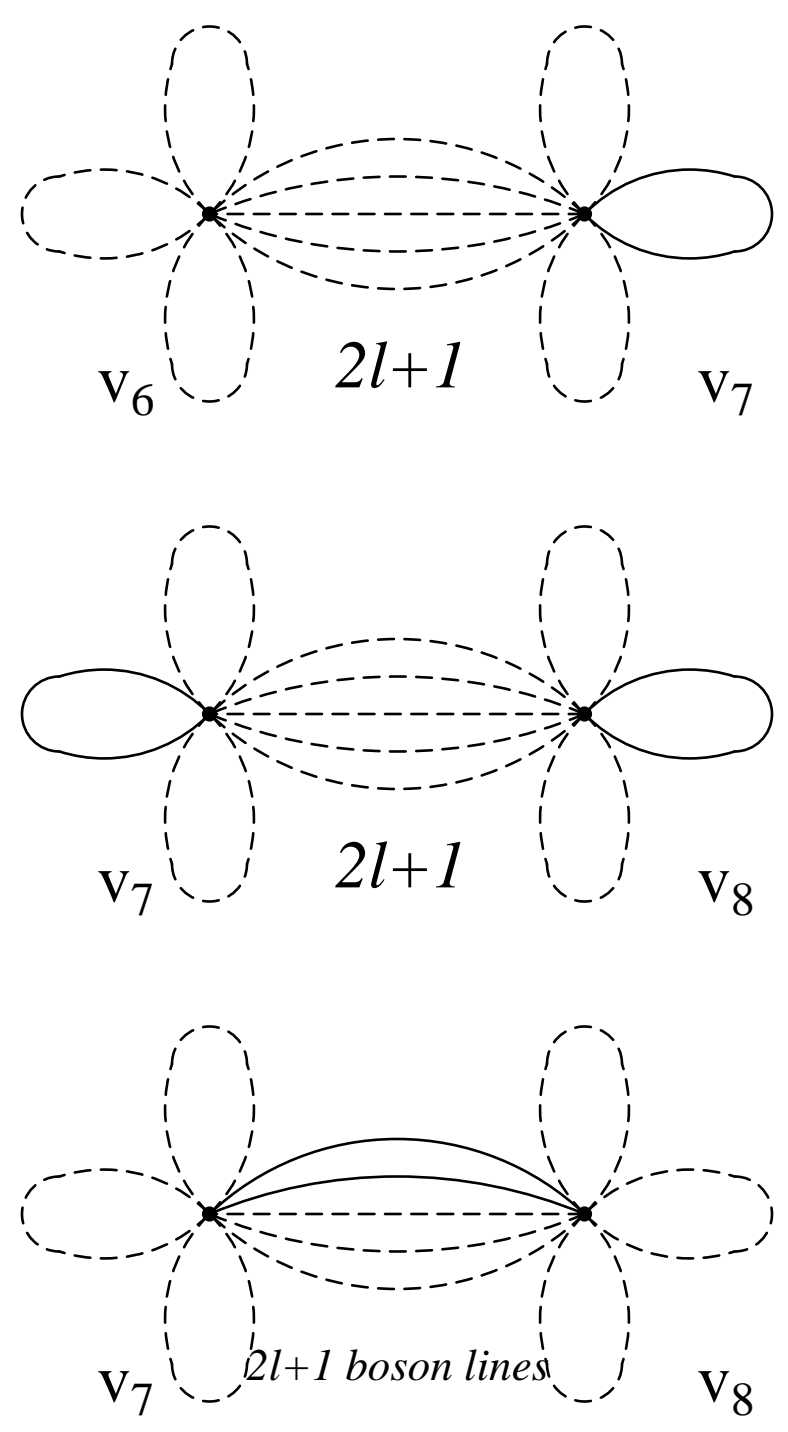

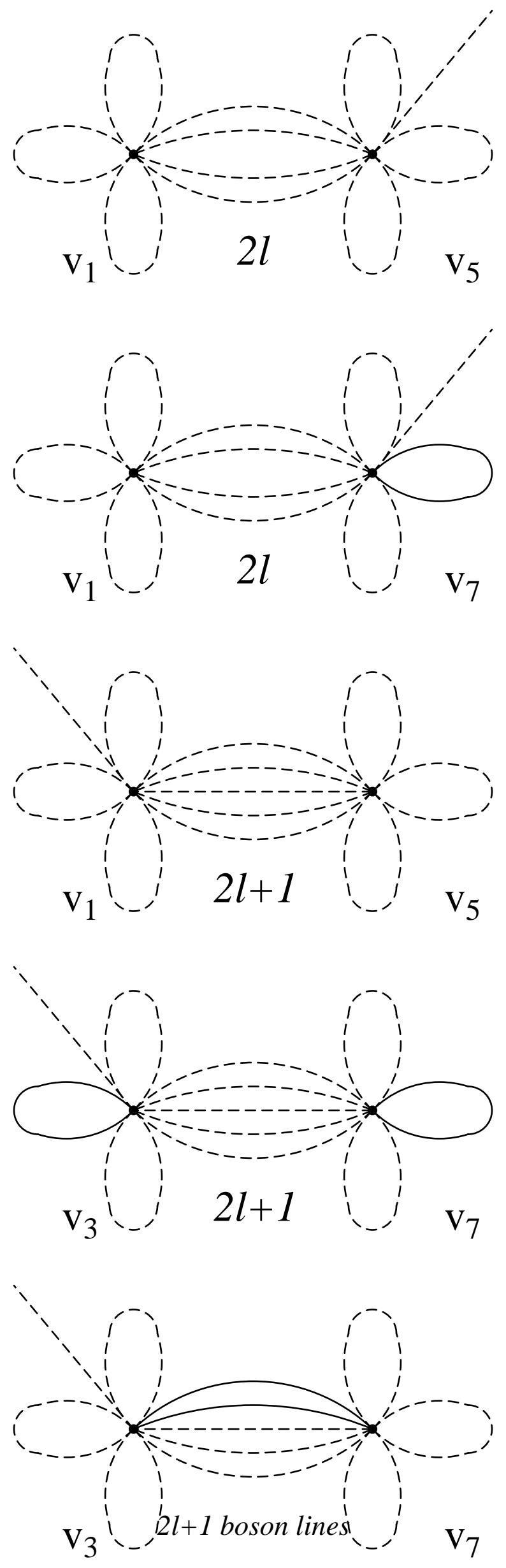